\title{
TESTS OF THE RADIAL TREMAINE-WEINBERG METHOD
}

\author{
Sharon E. Meidt and Richard J. Rand \\ Department of Physics and Astronomy, University of New Mexico, Albuquerque, NM 87131 \\ Michael R. MERRIFIELD \\ University of Nottingham, School of Physics and Astronomy, University Park, Nottingham NG7 2RD, UK \\ Victor P. Debattista \\ Centre for Astrophysics, University of Central Lancashire, Preston PR1 2HE, UK \\ AND \\ Juntai SHEN \\ McDonald Observatory, University of Texas, Austin, TX 78712 \\ Received 2007 August 24; accepted 2007 November 26
}

\begin{abstract}
At the intersection of galactic dynamics, evolution, and global structure, issues such as the relation between bars and spirals and the persistence of spiral patterns can be addressed through the characterization of the angular speeds of the patterns and their possible radial variation. The radial Tremaine-Weinberg (TWR) method, a generalized version of the Tremaine-Weinberg method for observationally determining a single, constant pattern speed, allows the pattern speed to vary arbitrarily with radius. Here we perform tests of the TWR method with regularization on several simulated galaxy data sets. The regularization is employed as a means of smoothing intrinsically noisy solutions, as well as for testing model solutions of different radial dependence (e.g., constant, linear, or quadratic). We test these facilities in studies of individual simulations and demonstrate successful measurement of both bar and spiral pattern speeds in a single disk, secondary bar pattern speeds, and spiral winding (in the first application of a TW calculation to a spiral simulation). We also explore the major sources of error in the calculation and find uncertainty in the major-axis position angle most dominant. In all cases, the method is able to extract pattern speed solutions where discernible patterns exist to within $20 \%$ of the known values, suggesting that the TWR method should be a valuable tool in the area of galactic dynamics. For utility, we also discuss the caveats in, and compile a prescription for, applications to real galaxies.
\end{abstract}

Subject headings: galaxies: kinematics and dynamics — galaxies: spiral — galaxies: structure methods: numerical

\section{INTRODUCTION}

One of the prime unresolved issues in the dynamics and evolution of galaxy disks remains the origin and evolution of large-scale bar and spiral structure. Although the persistence of grand-design spirals has been tied observationally to the presence of bars or companions (Kormendy \& Norman 1979), virtually nothing is known about the actual lifetimes of spiral patterns. In addition, despite indications that the relation between bar and spiral pattern speeds (which Sellwood \& Sparke [1988] first argued might not be equal) may be important for understanding the role of bars in angular momentum transfer during secular disk evolution (e.g., Debattista \& Sellwood 1998, 2000), there are as yet unanswered questions about the connections between multiple patterns in different radial zones. While mode coupling between patterns, which allows efficient outward angular momentum transfer in disks (Sygnet et al. 1988; Masset \& Tagger 1997), seems a most promising link, in two-dimensional (2D) $N$-body simulations with a dissipative gas component Rautiainen \& Salo (1999) find evidence for spiral structure in the absence of a bar, bar-spiral mode coupling, spiral-spiral mode coupling, and multiple pattern speeds without mode coupling.

Clearly, to address questions about the persistence of spiral patterns and the relation between bars and spirals requires not only determination of the pattern speed but how it varies with radius; only with accurate measurement of bar and spiral or inner and outer spiral pattern speeds in the same galaxy can we confirm whether spiral structure is steady or winding, whether bars and spiral pattern speeds are equal or unrelated, whether mode coupling exists, and the domain and number of patterns that can be sustained in a disk.

Because they are not directly accessible through observation, pattern speeds are often determined with indirect means such as the identification of predicted behavior at resonance radii (e.g., Elmegreen et al. 1989, 1996) or kinematic and morphological comparisons of simulated and observed structure (e.g., Rautiainen \& Salo 2005; Garcia-Burillo et al. 1993). It is also clearly desirable to employ methods for estimating pattern speeds that do not rely on theoretical models or simulation. Many other pattern speed determinations have therefore centered on the use of the model-independent method of Tremaine \& Weinberg (1984; hereafter TW), which presents a rigorous derivation for the pattern speed $\Omega_{p}$ based on the requirement of continuity using observationally accessible quantities. The determination of $\Omega_{p}$ involves surface density-weighted position and velocity line integrals parallel to the galaxy major axis under several essential assumptions. Specifically, the method requires that the disk of the galaxy is flat (unwarped) and contains a single, well-defined rigidly rotating pattern; that the surface density of a kinematic tracer of a disk component, which must obey continuity, becomes negligibly small at some radius and all azimuths within the map boundary (thereby critically yielding converged integrals); and that the relation between the emission from this component and its surface density is linear, or if not, suspected deviations from linearity can be modeled. 
In that information from all sampled radii is associated with a single, constant pattern speed, the TW calculation poses a challenge for extracting multiple distinct or radially varying pattern speeds. Nonaxisymmetric structures beyond a dominant pattern such as a bar in the disk of a galaxy will interfere with the measurement of $\Omega_{p}$ of the bar; when a nonaxisymmetric disk can be decomposed into two components with different pattern speeds, then the TW estimate is a luminosity- and asymmetry-weighted average of the two patterns (Debattista et al. 2002b). The TW estimate for the secondary pattern speed in the inner disk of NGC 6946 observed in $\mathrm{H} \alpha$, for example, is estimated to be limited to an uncertainty of as much as $50 \%$ given the primary pattern's contribution to the TW integrals (Fathi et al. 2007).

Such issues notwithstanding, there have been several recent adaptations of the TW method to positive effect. Applications of the TW method to SB0 galaxies using stellar light as a tracer extend the limits of integration in the TW calculation to just past the end of the bar in order to minimize contributions to the TW integrals from nonaxisymmetric (and several magnitudes dimmer) features beyond the bar; in such cases, integrating past the structure of interest is found to be sufficient for achieving converged integrals. The bar pattern speeds in NGC 7079 (Debattista \& Williams 2004) and NGC 1023 (Debattista et al. 2002a), for example, have both been successfully measured in this way.

When there exists more than one pattern in distinct radial zones, however, arguments about the convergence of the TW integrals are less straightforward. To measure the secondary bar pattern speed in NGC 2950, Corsini et al. (2003) and Maciejewski (2006) explore decoupling the inner secondary and outer primary bar pattern speeds by associating each component with unique surface brightness contributions in the TW calculation. The TW integrals are modified by the presence of the inner pattern based on an assumption about how and where the two patterns decouple (to first order). This analysis has confirmed the existence of, if not measured, a unique secondary bar pattern speed possibly indicating counterrotation with respect to the primary bar.

At their best, improving the accuracy of pattern speed estimates to about $20 \%$ (Gerssen \& Debattista 2007), these kinds of adaptations of the TW method for measurement of single or multiple patterns still require assumptions about bar extent based on morphological or kinematic signatures. To separate the observed surface brightness in NGC 2950 into secondary and primary bar components, Maciejewski (2006) must assume that the patterns indeed decouple at the inner bar end, or that the outer pattern is axially symmetric at least within the inner pattern's extent. There, the transition between the two is inferred from the location of a plateau in the TW surface brightness-weighted position integral as the limits of integration are extended from zero. Perhaps more critically, as investigated in $\S 4.2$, the direct association of bar length measured in this manner (or perhaps others) with pattern extent may introduce error into TW calculations.

Furthermore, adaptations of the TW method based on this type of identification are likely to be inapplicable for spiral pattern estimation. In spiral galaxies, not only can identifying transitions between patterns be less clear, but nonaxisymmetric motions are significantly smaller (e.g., Roberts \& Stewart 1987) than in bars (at least those typically analyzed with the TW method). Indeed, the TW method has yet to be tested on a simulated spiral.

A recent modification to the TW calculation (Merrifield et al. 2006, hereafter MRM06) in which $\Omega_{p}$ is allowed radial variation promises to be an invaluable resource for tests of long-lived density wave theories and for understanding the connection, if any, between bar and spiral pattern speeds. Like the TW method, the so-called radial TW (TWR) method uses measurements of ob- servables to extract radially varying pattern speeds. As first applied using the BIMA Survey of Nearby Galaxies CO observations of the grand-design Sb galaxy NGC 1068 (MRM06), the TWR method returned a spiral pattern speed solution that declines with radius, allowing a winding time for the pattern to be estimated (e.g., MRM06).

As described in MRM06, the nature of the discretized calculation presents numerical solutions that are highly susceptible to fluctuations as a result of compounded noise in the data. In this paper $(\S 2.2)$ we develop the TWR method with regularization as a means of smoothing intrinsically noisy solutions, as well as testing model solutions of different radial dependence (described in $\S 2.3$ ). These and other commendations notwithstanding, with regularization one may risk introducing an unrealistic prejudice to TWR solutions. In $\S 3.1$ we address a means of identifying when this is likely to occur, and in $\S 3.2$ we describe a scheme for minimizing such regularization-induced bias.

Using evidence that arises from these considerations, as well as other a priori information, theoretically and observationally motivated models for $\Omega_{p}(r)$ can be developed that constrain the number and extent of patterns present in a disk. Once solutions for these models are calculated, the goodness of each must be assessed in order to identify the best-fit solution. In $\S 3.3$ we outline the criteria with which the models are judged and describe our concept of error evaluation in the final solutions.

Beginning in $\S 4.1$, we analyze three simulated galaxies with known pattern speeds (a barred spiral, a slowly winding spiral, and a double-barred spiral, marking the first application of the TW method to simulated spiral patterns) in order to develop a general stratagem for application to real galaxies. As applied to these simulations, we find that the TWR method is able to extract multiple pattern speeds with accuracies on the order of (and in some cases better than) the traditional TW method. With the barred spiral simulation in $\S 4.2$ we show that TWR bar pattern speed measurement presents an improvement over traditional TW bar estimates, particularly when there is evidence of a significant contribution from the spiral pattern to the TW integrals. We find that the regularized TWR method can recover information from both patterns effectively by identifying and treating the bar-tospiral transition radius (which the TW values themselves may not indicate) as a free parameter in the calculation. In $\S 4.2 .1$ we analyze the results of the method in detail, particularly with regard to morphological limitations. We compare our TWR results with TW estimates in $\S 4.2 .2$ and examine the influence of systematic errors due to the assumed disk position angle and inclination (shown to be crucial for TW estimates in Debattista 2003) on both in $\S 4.2 .3$. We also explore the reliability of a fixed parameterization for the bar-to-spiral transition radius $(\S 4.2 .4)$.

In the last third of the paper, we investigate the prospects for extracting spiral pattern speed solutions that are winding in nature ( $\S 4.3)$, marking the first application of the TW method to a spiral simulation, and in $\S 4.4$ we address the use of the TWR method for the purposes of parameterizing an independently rotating nuclear bar in a double-barred simulation. There, the techniques we employ for decoupling and extracting measurements of the pattern speeds of both the primary and secondary bar components may present an interesting corollary to recent attempts with the TW method to measure secondary bar pattern speeds in the presence of a strong primary bar pattern. We note here that the TWR method is a generalized version of the procedure used on NGC 2950 (see Corsini et al. 2003; Maciejewski 2006); separating the surface brightness into two components can be thought of as the coarsest version of the discretization that is the backbone of numerical TWR solutions. 
Based on our experience with these simulations, we conclude with comments on the applicability of the method to observations of real galaxies in $\S 5.1$, where we also outline a general prescription for using the TWR calculation with regularization.

\section{THE TWR METHOD WITH REGULARIZATION}

\subsection{The Radial Tremaine-Weinberg Method}

By proceeding under the aforementioned assumptions of Tremaine \& Weinberg (1984), but allowing that $\Omega_{p}$ may possess spatial variation in the radial direction whereby the surface density of the chosen tracer can be written $\Sigma(x, y, t)=\Sigma\left(r, \phi-\Omega_{p}(r) t\right)$, with appropriate mathematical generalizations, the derivation and measurement of the pattern speed $\Omega_{p}(r)$ can be made from observable intensities and kinematics of a chosen tracer.

Following the derivation given in MRM06, integrating the continuity equation obeyed by the tracer [with the replacement $\left.\partial \Sigma / \partial t=-\Omega_{p}(r) \partial \Sigma / \partial \phi\right]$

$$
-\Omega_{p}(r) \frac{\partial \Sigma}{\partial \phi}+\frac{\partial \Sigma v_{x}}{\partial x}+\frac{\partial \Sigma v_{y}}{\partial y}=0
$$

over $x$ and $y$ (thereby eliminating the unobservable $v_{x}$ and the spatial derivative $\partial / \partial y$ ), and changing from Cartesian to polar coordinates yields

$\int_{r=y}^{\infty} \int_{\phi=\arcsin (y / r)}^{\pi-\arcsin (y / r)} \Omega_{p}(r) \frac{\partial \Sigma}{\partial \phi} r d r d \phi+\int_{-\infty}^{\infty} \Sigma v_{y} d x=0$.

A final integration with respect to $\phi$ results in a Volterra integral equation of the first kind for $\Omega_{p}(r)$ :

$$
\int_{r=y}^{\infty}\left\{\left[\Sigma\left(x^{\prime}, y\right)-\Sigma\left(-x^{\prime}, y\right)\right] r\right\} \Omega_{p}(r) d r=\int_{-\infty}^{\infty} \Sigma v_{y} d x
$$

where $x^{\prime}(r, y)=\left(r^{2}-y^{2}\right)^{1 / 2}$.

Note that with constant $\Omega_{p}$ in equation (3), we arrive at the regular TW result

$$
\Omega_{p} \int_{-\infty}^{\infty} \Sigma x d x=\int_{-\infty}^{\infty} \Sigma v_{y} d x
$$

which, with normalization by $\int \Sigma d x$ (e.g., Merrifield \& Kuijken 1995), leads to

$$
\Omega_{p}=\frac{\langle v\rangle}{\langle x\rangle},
$$

where $\langle v\rangle=\int \Sigma v_{y} d x / \int \Sigma d x$ and $\langle x\rangle=\int \Sigma x d x / \int \Sigma d x$.

For a galaxy projected onto the sky plane with inclination $\alpha$ (so as to distinguish from index $i$ ), both the kernel on the left and the integral on the right of equation (3) are observationally determined quantities with $x=x_{\mathrm{obs}}, y=y_{\mathrm{obs}} / \cos \alpha$, and $v_{y}=v_{\mathrm{obs}} /$ $\sin \alpha$, where $x_{\text {obs }}$ and $y_{\text {obs }}$ are the coordinates in the plane of the sky along the major and minor axes, respectively, and $v_{\text {obs }}$ is the observed line-of-sight velocity. Solutions can be extracted numerically by replacing the integral on the left with a discrete quadrature for different values of $y=y_{i}$ and $r=r_{j}$ (see Fig. 1), whereby equation (3) is converted to

$$
\Sigma_{r_{j}>y_{i}} K\left(y_{i}, r_{j}\right) \Omega_{p}\left(r_{j}\right)=b\left(y_{i}\right)
$$

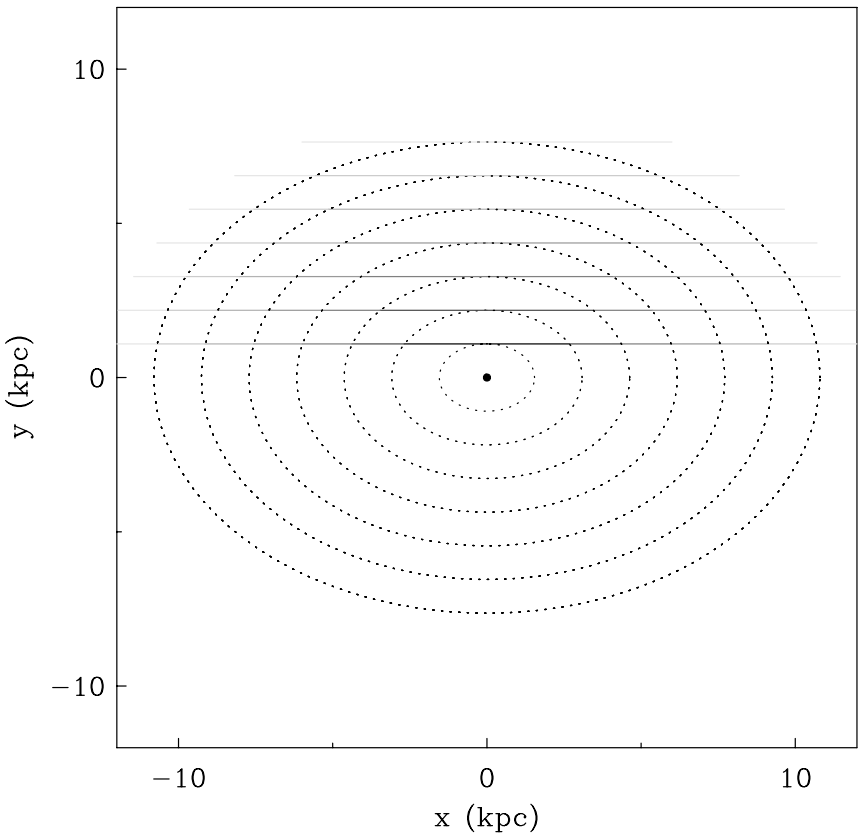

FIG. 1.-Illustration of a $y>0$ quadrature for a galaxy viewed from the negative $z$ side with a tilt around the $y$-axis by $\alpha=45^{\circ}$. The horizontal lines, or slices, at positions $y_{i}$ are spaced at $\Delta y=1.54$, or $\Delta y_{\mathrm{obs}}=1.09$, and represent integration between the limits $\pm\left(R_{0}^{2}+y_{i}^{2}\right)^{1 / 2}$, where $R_{0}=10.8$ is the maximum radial extent of the quadrature. Each slice is carved into elements of width $\Delta r$ whereby all the elements with the same shade of gray represent a single radial bin $r_{j}$.

or to a matrix equation of the form

$$
K_{i j} \Omega_{j}=b_{i}
$$

with $\boldsymbol{K}$ an upper triangular $N \times N$ square matrix. Note that numerical quadratures on either side of the galaxy $(y<0$ or $y>0)$ occur independently, providing two measures of $\Omega_{p}(r)$. Furthermore, as governed by the information available, the slices that delimit the quadrature on a single side need not be uniformly spaced. In this case, solutions inherit a variable bin width $\Delta r$. Also, the calculation allows for no azimuthal dependence for the pattern speed, which we assume throughout.

The size of $\boldsymbol{K}$ depends on the desired coarseness or fineness of the quadrature; the separation between slices at positions $y_{i}$ (limited by either the resolution or the sampling of the data) translates into a radial bin width (modulo $\cos \alpha$ ) via equation (7). The quadrature, perhaps more critically, depends on the limits of integration in equation (3). These limits $\pm X_{\max }$ should be chosen based on where the integrals have converged. While in the case of a single bar pattern integrating past the structure of interest is often suitable, as shown in Zimmer et al. (2004, Figs. 9-11), in the presence of strong, extended asymmetry TW values are highly dependent on the extent of integration along each slice $i$. In cases where multiple patterns exist in a single disk, then, it is equally favorable (and hopefully sufficient) to extend all integrals to the edge of the surface brightness distribution.

Meeting the requirement of integral convergence in this manner as applied to the TWR calculation determines the location of the last radial bin $j_{\max }$ associated with elements $K_{i j_{\max }}$ along each slice. For a given radial bin width, with the requirement that $j_{\max }$ equals $N$ we are presented with the size of $\boldsymbol{K}$, as well as the outermost slice position, since $j_{\max }$ must also equal $i_{\max }$. One should check to see that $K_{N N}$, the last entry in $\boldsymbol{K}$ associated with 

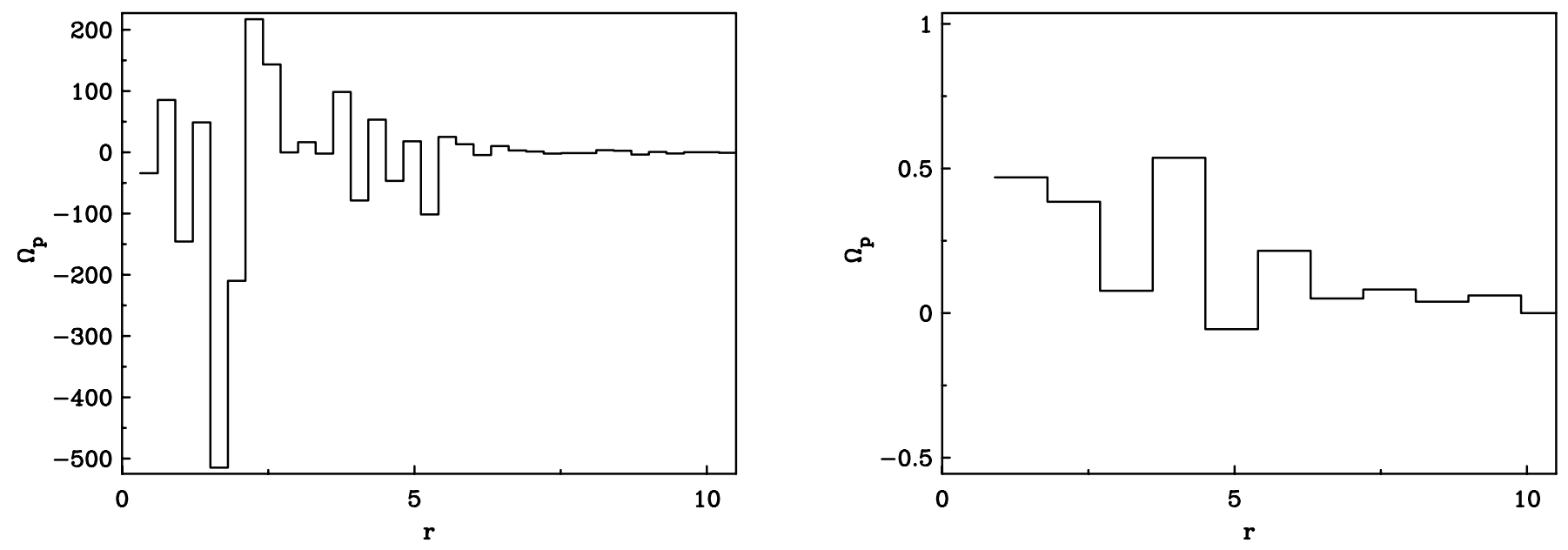

FIG. 2.-Plots of (unregularized) TWR solutions for two different binnings of data from the barred spiral simulation in $\S 4.2$ with SA $=-45^{\circ}$ (see $\S 4.1$ for orientation convention). The left (right) panel shows the solution generated using $\Delta r=0.3(0.9)$ bins.

the outermost slice, is associated with a fully converged $\int \Sigma x d x$ (which achieves convergence at least by the map boundary).

Since $\boldsymbol{K}$ is an upper triangular matrix, the $\Omega_{j}$ can be solved for via simple back substitution. In this way, solutions are generated from the outermost to the innermost radius (from light gray to black in Fig. 1) according to

$$
\Omega_{k}=\frac{b_{i}-\sum_{j>k}^{N} K_{i j} \Omega_{j}}{K_{k k}},
$$

with $k \geq i$ (from eq. [7]). Such solutions, however, are especially susceptible to wildly oscillatory behavior as errors from large radii propagate inward. These compounded errors arise from uncertainties in the velocities and intensities (which get translated into the $K_{i j}$ ) and can be particularly severe since the outermost bins often cover the lowest signal-to-noise ratio regions in the data. The errors thus introduced can be systematic.

Figure 2 shows an example of (unregularized) TWR solutions for two different binnings using data from the barred spiral simulation of $\S$ 4.2. By increasing the bin width, the largest oscillations are reduced, but (as will become clearer) even tripling the bin width will not necessarily limit the propagation of noise to the level required for the extraction of realistic solutions. (A more thorough discussion of the implications for TWR solutions is deferred until the beginning of $\S$ 4.2.)

Although the initial application of the TWR method on a real galaxy, namely, NGC 1068 (MRM06), showed little of the oscillatory behavior common to noisy, discretized Volterra-type solutions (outside $r \sim 1.5 \mathrm{kpc}$, anyway), the solutions were generated over relatively few bins (only five at most, over the region $r=$ $1.5-2.8 \mathrm{kpc}$ ). In general, while large bin widths can often minimize the propagation of noise in the calculation, they can be expected to compromise solutions, as is discussed in the sections to follow; naturally, the smaller the quadrature element, the more accurately the true radial variation of the pattern speed can be ascertained. This is particularly critical as applied to disks sustaining multiple patterns where, as described later, a certain degree of radial precision is required for accurate separation of the pattern speeds.

Barring a large, limiting resolution, one should expect to be able to perform a sufficiently smooth quadrature wherein the number of elements in $\boldsymbol{K}$ becomes large. Since more elements in $\boldsymbol{K}$ (and more bins over which to generate solutions) result in intrin- sically noisy behavior, an effect most pronounced in the innermost bins, gaining a finer, more accurate quadrature often means forfeiting control of the solution. By combining regularization with the TWR calculation to force a smooth solution, however, one can counter this effect while maintaining the required precision.

Regularization also serves to alleviate the impact of nonglobal features that are most likely not included in the overall pattern (and which can singularly introduce large errors into the integrals). Since rapid fluctuations in $\Omega_{p}(r)$ are penalized, discrepant points need not be avoided or ignored (as demanded in performing the TWR calculation on NGC 1068 in MRM06). While one may also use the alternative, which would be to fit models of $\Omega_{p}$ directly to equation (7) and perform a grid search to find the best model form and coefficients, we pursue regularization here, its speed making it preferred.

\subsection{Regularization}

Our procedure entails the following. As a modification to the $\chi^{2}$ estimator minimized by solutions $\Omega_{j}$ of equation (7), namely,

$$
\frac{\left|K_{i j} \Omega_{j}-b_{i}\right|^{2}}{\sigma_{i}^{2}},
$$

with implicit sum over $i$ (and $j$ ) and errors $\sigma_{i}$ representing the measurement error of the $i$ th data point $b_{i}$, we introduce a regularizing operator, or smoothing functional $S$, containing a priori information in the manner of Tikhonov-Miller regularization (Tikhonov \& Arsenin 1997; Miller 1970) in which (in matrix form) solutions $\Omega$ minimize

$$
|\overline{\boldsymbol{K}} \cdot \boldsymbol{\Omega}-\overline{\boldsymbol{b}}|^{2}+\lambda \boldsymbol{\Omega} \cdot \boldsymbol{S} \cdot \boldsymbol{\Omega} .
$$

Here the elements of $\overline{\boldsymbol{K}}$ and $\overline{\boldsymbol{b}}$ are $K_{i j} / \sigma_{i}$ and $b_{i} / \sigma_{i}$, respectively, and the role of $\lambda$, controlling the relative amount of $\chi^{2}$ minimization on the left to entropy maximization on the right, is explicit.

Reduced to a linear set of normal equations, this minimization returns smoothed solutions according to a modified version of equation (7):

$$
\left(\overline{\boldsymbol{K}}^{T} \cdot \overline{\boldsymbol{K}}+\lambda \boldsymbol{S}\right) \cdot \boldsymbol{\Omega}=\overline{\boldsymbol{K}}^{T} \cdot \overline{\boldsymbol{b}}
$$


Note that the regularizing functional, not necessarily upper triangular, introduces an anticipatory quality to solutions $\Omega_{j}$ whereby all bins at the same radius are coupled. Furthermore, solving for components $\Omega_{j}$ no longer only involves a procedure like back substitution, but requires rather an L-U decomposition (for instance) as well.

\subsection{The Smoothing Operator}

The real power in applying regularization to TWR calculations is in the freedom to choose how the smoothness of solutions is achieved. For the purposes of distinguishing between different possible radial dependencies for $\Omega(r)$, we choose $S$ to reflect a priori assumptions based on simple expectations from theory and observation. Model solutions, then, each incorporating its own $S$, represent smoothed, testable realizations of the pattern speed. These we restrict to simple forms in order to minimize the additional amount of information to be extracted from the data relative to the traditional TW method.

For polynomial solutions, we consider only constant, linear, and quadratic radial dependence. The elements of the smoothing $\boldsymbol{S}$ are associated with the minimization of the $n$th derivative of $\Omega(r)$ for each polynomial solution of order $n$. For instance, for linear solutions this entails minimizing

$$
\boldsymbol{\Omega} \cdot \boldsymbol{S} \cdot \boldsymbol{\Omega}=\sum_{n=1}^{N-2}\left|-\Omega_{n}+2 \Omega_{n+1}-\Omega_{n+2}\right|^{2},
$$

whereupon

$$
\boldsymbol{S}=\left(\begin{array}{ccccccccc}
1 & -2 & 1 & 0 & 0 & 0 & 0 & \ldots & 0 \\
-2 & 5 & -4 & 1 & 0 & 0 & 0 & \ldots & 0 \\
1 & -4 & 6 & -4 & 1 & 0 & 0 & \ldots & 0 \\
\vdots & & & & \ddots & & & & \vdots \\
0 & \ldots & 0 & 1 & -4 & 6 & -4 & 1 & 0 \\
0 & \ldots & 0 & 0 & 1 & -4 & 6 & -4 & 1 \\
0 & \ldots & 0 & 0 & 0 & 1 & -4 & 5 & -2 \\
0 & \ldots & 0 & 0 & 0 & 0 & 1 & -2 & 1
\end{array}\right) .
$$

One may also choose a form for $\boldsymbol{S}$ that identifies two or more distinct regions of independent radial behavior by invoking a step function model. For the case of a barred spiral with a constant bar and quadratic spiral, for instance, this corresponds to minimizing

$$
\boldsymbol{\Omega} \cdot \boldsymbol{S} \cdot \boldsymbol{\Omega}= \begin{cases}\sum_{n=1}^{t-1}\left|\Omega_{n}+\Omega_{n+1}\right|^{2}, & n<t \\ \sum_{n=t}^{N-3}\left|-\Omega_{n}+3 \Omega_{n+1}-3 \Omega_{n+2}+\Omega_{n+3}\right|^{2}, & n \geq t .\end{cases}
$$

The elements of $S$ with $n<t$ reflect the a priori assumption that the bar pattern speed is constant, while those for $n>t$ associate a quadratically varying pattern speed with the spiral. The index $t$, a free parameter, locates the radial bin where the transition between the two patterns occurs. Obviously, the number of available bins constrains the order of the polynomial in a given radial zone.

Once we have chosen $S$, we initially choose $\lambda$ to reflect comparable amounts of $\chi^{2}$ minimization and regularization by letting $\lambda=\lambda_{0}=\operatorname{Tr}\left\{\overline{\boldsymbol{K}}^{T} \cdot \overline{\boldsymbol{K}}\right\} / \operatorname{Tr}\{\boldsymbol{S}\}$. Since we are in the business of generating solutions based on particular models, $\lambda$ is modified to arrive at the regularization required to return solutions of a given type. This modification generally consists of an increase in $\lambda$ over $\lambda_{0}$. Consider how the regularizing parameter $\lambda$ regulates the degree of smoothness of the solution to the weight placed on the data: with $\lambda=0$, equation (10) corresponds to $\chi^{2}$ minimization (and becomes an unbiased estimator with the smallest variance), however yielding highly oscillatory solutions, while $\lambda \rightarrow \infty$ corresponds to a maximally smooth estimator with nonvanishing variance.

Fitting data sets with different spatial coverage will change the effect of $\lambda$ on the solution (e.g., larger bins require less regularization). The most appropriate choice for $\lambda$ (and $\boldsymbol{S}$ ) should be made on a galaxy-by-galaxy basis, according to the quality of information to be extracted from observations.

\section{OTHER CONSIDERATIONS}

\subsection{Regularization-induced Bias}

By imposing assumptions about the smoothness of the pattern speed, regularization inevitably introduces complications for extracting realistic solutions. To understand how these arise, consider the solution for a barred spiral galaxy. The nature of the calculation (from out to in) has implications for the accuracy of the bar estimates, in particular. Not only do the $\Omega_{j}$ for bins covering the bar rely on the greatest number of matrix elements (and errors therein), but the bar estimate depends critically on the solution for the spiral and all outer bins via equation (8). Consequently, merely requiring the pattern speed in the outer bins to be constant with regularization out to the edge of the surface density (for instance), effectively removing fluctuations that might better fit the data, will have consequences for the bar solutions. So while the regularization is particularly fast and effective for tests for the radial behavior of patterns, it can also hinder the realization of accurate solutions.

For the simulations studied here, the risk of regularizationinduced bias is inherited from the adopted quadrature. Recall our requirement that all slices cover the full extent of the "emission," so as to ensure all integrals be fully converged, and relatedly, that the last matrix element governs the outermost slice position. In the barred spiral simulation of $\S 4.2$, for example, such an extensive quadrature presents us with outermost slices that pass through a region where there is simply no discernible pattern (as indicated by the surface brightness distribution and its Fourier decomposition; see next section). While these slices themselves do not provide direct estimates of the patterns of interest, the corresponding bin values are necessary for calculating the bar and spiral solutions. Moreover, the quality of these solutions will be intimately related to the treatment of the outermost bins. For accurate pattern speed measurement, we therefore find it essential to identify, and reduce the influence of, the compromised zone by not enforcing regularization on these bins.

\subsection{Fourier Diagnostics}

With the above concerns in mind, we have tested and used the following scheme. Given slices that pass through an outer region that either contains little information from a strong pattern, is suspected of sustaining multiple patterns, or displays only faint emission, we choose in such a case to let the values in the outermost bins be calculated without regularization with the restriction, only, that they minimize the $\chi^{2}$. Once a particular bin (at $r_{c}$, the cut radius) has been reached, regularization is imposed with all remaining inward bins generated accordingly. 


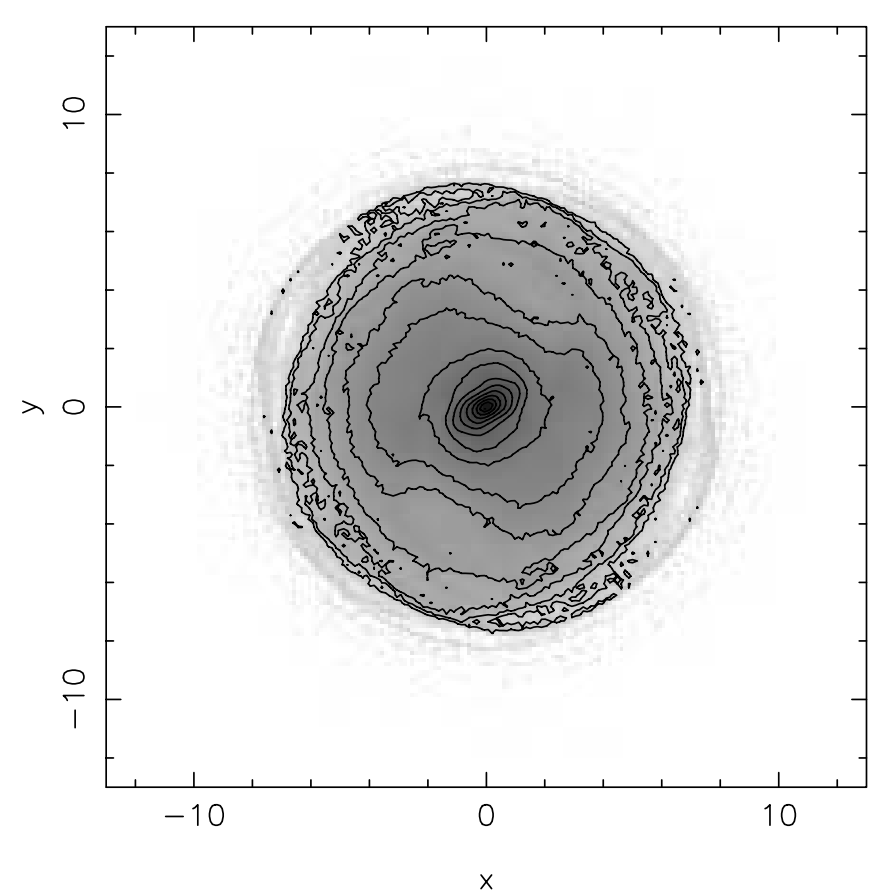

FIG. 3.-Face-on display of the barred spiral simulation's surface brightness distribution projected with a $-30^{\circ}$ rotation about the $z$-axis. For reference, the alignment of the TWR quadrature for a frame at this orientation is designated $\mathrm{SA}=+60^{\circ}$.

In our procedure this corresponds to using an $S$ indexed by the cut bin $c$ that is lowest block zero. And the "cut" radius identifies the location in the disk where the outermost discernible pattern ends. Note that this is in contrast to altogether ignoring the outer portion of the disk. We prefer this procedure for two reasons: (1) as qualified later in $\S 4.2 .2$, when each integral is truncated within the disk, the quadrature is at greater risk of ignoring information critical for characterizing the patterns uniformly throughout the disk; and (2) like the transition radius $r_{t}$, we can easily incorporate $r_{c}$ as a free, although restricted, parameter in our models.

In practice, extracting the bar and spiral pattern speeds for the simulated barred spiral in $\S 4.2$ involves generating a group of solutions with various bar-to-spiral pattern transition radii for a given cut location. Throughout the analysis, we choose the cut radius to reflect a priori knowledge of the outermost measurable pattern's termination radius estimated from the surface brightness and its Fourier decomposition. When referred to, the power in each Fourier component, or mode $m$, is given by the norm of the complex Fourier amplitude

$$
I_{i}=\sum_{n=1}^{N} e^{i m \theta_{n}}
$$

where $\theta_{n}$ is the angular coordinate of each of the $N$ particles at each measured radius.

For the barred spiral in $\S 4.2$, for example, we combine evidence from the surface density (Fig. 3), where beyond the bar there is enhanced spiral surface density from only a limited radial zone, with the Fourier spectrum to identify a region in the disk outside the spiral that is susceptible to regularization-induced bias. Specifically, the Fourier power spectrum (Fig. 4) shows that at $r \sim 5.0$ the second clean hump in $m=2$ power decreases to almost zero, marking the end of the spiral. Past this radius, the (strong) $m=2$ component (between $r \sim 6.0$ and 8.0) is not associated with

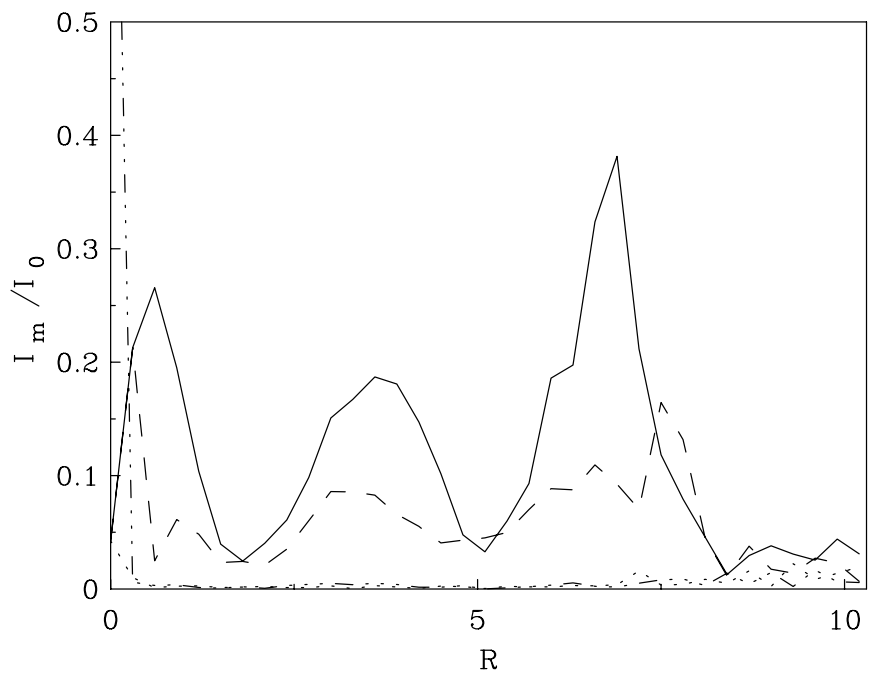

FIG. 4.-Fourier power spectrum of the barred spiral simulation's surface brightness distribution shown in Fig. 3. Modes up to $m=4$ are plotted as a function of radius: $m=1$ (dotted line), $m=2$ (solid line), $m=3$ (triple-dot-dashed line), and $m=4$ (dashed line).

visible spiral structure (see Fig. 3). Reckoning this outer zone to be incompatible with a simple pattern speed model, then, we consider only the inward bar and primary spiral pattern speeds to be measurable with regularization. Figure 19 of Debattista et al. (2006, hereafter D06) for this simulation (Fig. 5 in this paper) confirms this; not only does the spiral pattern terminate at $r_{c} \sim 5.0$, but beyond this radius the pattern speed is multivalued (this, of course, would be indiscernible in a real galaxy). In this case, imposing form with regularization on bins of suspect quality and behavior outside the spiral will likely impair the solution of interest. We therefore restrict the cut bin for the barred spiral simulation to $4.5<r_{c}<6.0$, representative of where the primary spiral pattern terminates in the disk. As mentioned in $\S 4.2$, this step is substantiated by our finding that a cut radius of $r_{c}=4.8$ is one of several $\chi^{2}$ minima given a range of possible cut radii. And furthermore, solutions generated in this manner are judged to overall provide a considerably better fit to the data than solutions where regularization is imposed out to the edge of the surface brightness (according to the scheme described in the next section).

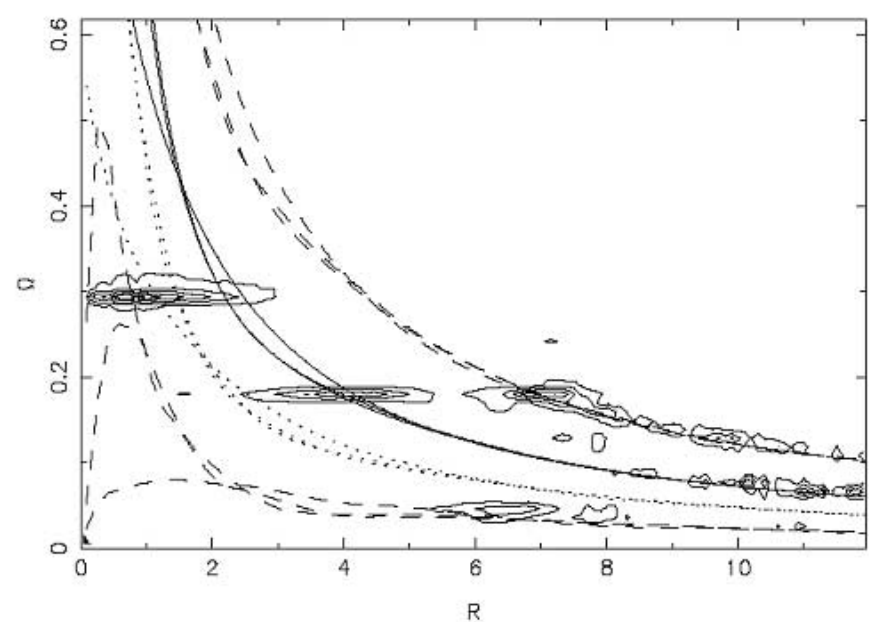

FIG. 5.-Contours of the barred spiral simulation's $m=2$ Fourier mode showing a bar pattern speed $\Omega_{b}=0.29$, a bar-to-spiral transition of $r_{t} \sim 2.5$, and a dominant spiral pattern speed $\Omega_{s}=0.18$ out to $r \sim 4.5-5.5$, beyond which multiple spiral modes exist. 


\subsection{Weighting Schemes and Goodness of Fit}

Given the data, we simultaneously generate model solutions with different radial dependencies for direct comparison for each side $(y>0$ or $y<0)$ independently. We then average the two like-model solutions together to construct a global solution. (In those instances when model solutions include a cut bin, the averaging occurs over the regularized part of the solutions only, in order to maintain the "unbiased" quality of the unregularized part of the solutions for each side of the galaxy.) Note that while the assumption that the patterns are indeed global is not overly inspired for the simulated galaxies studied here, putting this into practice on a real galaxy requires that the assumed galaxy kinematic parameters are accurate and that such symmetry exists.

With each global model solution we generate a complete set of $\langle v\rangle$ using equation (7). We then judge each model through the $\chi_{\nu}^{2}$ ( $\chi^{2}$ per degree of freedom) goodness-of-fit estimator of the reproduced to actual $\langle v\rangle_{i}=b_{i} /\left(\int \Sigma d x\right)_{i}$ given measurement errors $\sigma_{i}^{\langle v\rangle}$, in keeping with the standard TW analysis. Note that with this choice the $\sigma_{i}$ in equation (9) are related to the errors $\sigma_{i}^{\langle v\rangle}$ by $\left(\int \Sigma d x\right)_{i}$. That is, the calculation fits to the $b_{i}$ given errors $\sigma_{i}$ while our $\chi^{2}$ estimator considers the differences from $\langle v\rangle_{i}$ given errors $\sigma_{i}^{\langle v\rangle}$.

While for a real galaxy inaccuracies in the assumed position angle (PA) have the largest potential for introducing errors into $\langle v\rangle_{i}$, we prefer that the measurement errors $\sigma^{\langle v\rangle}$ reflect random noise in the data, only. (Systematic errors prove more practically assessed through direct tests of the sensitivity of the results to departures from the nominal values for the PA or inclination, for instance.) For the simulations studied here, then, we obtain errors $\sigma_{i}^{\langle v\rangle}$ under the assumption that the inverse mirror image of each $\langle v\rangle_{i}$ on one side of the galaxy should be the same as on the other side (i.e., the patterns are symmetric). We then assign a global error $\sigma^{\langle v\rangle}$ to each slice where $\sigma^{\langle v\rangle}=\left(\sum_{i=1}^{2 N} \sigma_{i}^{\langle v\rangle}\right) / 2 N$ (and $N$ is the number of bins/slices used in the TWR calculation on a single side).

In practice, the simplest $\chi^{2}$ weighting schemes are either uniform weighting for all slices or weighting by the intensity, which should give more weight to slices where the signal is strongest. We have chosen the former since we are interested in $\Omega_{p}$ over a broad radial range and prefer that our result is not dominated by just the slices with the highest signals (which can vary dramatically). Furthermore, the choice of assigning an identical error $\sigma^{\langle v\rangle}$ to each slice carries with it an implicit weighting scheme for equation (10). For an exponential surface brightness profile, for example, slices on either side of the galaxy will have progressively smaller $\int \Sigma d x$ as $\left|y_{i}\right|$ increases. This corresponds to errors $\sigma_{i}$ proportional to $\int \Sigma d x$, then, that grow larger from out to in. Since in most cases the uniform weighting scheme will be in actuality most restrictive of the outer bins (the goodness of which will affect the solution inward), this choice is particularly well suited for the TWR calculation.

Given our choice of weighting scheme, there are two important considerations that demand that we calculate the $\chi^{2}$ over all slices. First, the innermost bins contribute to the $\langle v\rangle$ in only the innermost slices and hence contribute relatively negligibly to the $\chi^{2}$, despite possibly larger weights $K_{i j}$ (reflective in part of a surface brightness that is centrally peaked, say) than bins at larger radius. This is especially true when an inner pattern appears only over a small fraction of the total bins, and the reproduced $\langle v\rangle$ of even those slices that pass directly through the inner pattern still rely (perhaps predominantly) on the solution out to the largest radial bin. However, since the goodness of the inner bins is directly related to the goodness of the bins at larger radii, by considering all slices we more effectively judge the whole solution.
Secondly, in cases when some number of outermost bins are calculated without regularization, it is critical to account for the (largely positive) effect that these bins have on the solution inward, especially from model to model. In our current scheme, the values of the "unregularized" bins are not quite identical to those in the completely unregularized solution since the quantity that they minimize still includes participation from nonzero elements in the smoothing functional $S$ and what is currently a modeldependent $\lambda$. That is, slight variation in the values of the unregularized bins from model to model is apparent, and we cannot ignore the minor differences this introduces to the regularized part of the solution. Although this is a minor effect, by considering the $\langle v\rangle$ of all slices in the $\chi^{2}$, we prevent solution preference based on the unregularized bins (which act essentially as standins) from being introduced.

The first consideration above also prevents us from calculating judicious error bars on the solutions according to the variation of individual model parameters over a typical $\chi^{2}$ confidence interval; in practice, the value of an inner pattern with minimal radial extent can change considerably with little effect on the $\chi^{2}$. Indeed, we find that the errors generated according to such a prescription are unrepresentative of the goodness of the solutions as returned by the calculation.

In $\S 4.2 .1$ we describe the dependence of both inner and outer speeds on the location assigned to the transition between the two. An obvious progression for future applications of the TWR method would be an exploration of the covariance of what we consider here, to first order, "free parameters," especially for the purposes of improved error estimation. Presently, however, we construct error bars for model solutions by considering the range of parameters in the best solutions at different assumed projections. As pertains to the sections that follow, by considering an overall solution in this manner, we can fairly account for the uncertainty introduced for real galaxies by the reality that each can be sampled at only a single PA.

\section{TESTS OF THE TWR METHOD ON SIMULATIONS}

In order to establish guidelines for applying the regularized calculation to observations of real galaxies, we next perform tests of the method on simulations with known pattern speeds. Each case invokes unique models for $\Omega_{p}(r)$, which we motivate and discuss in detail. The procedure for engaging the method with maximum accuracy then follows from careful examination of the quality of solutions given the available information. Although quite detailed, these individual studies together constrain general scenarios and practices to extrapolate onto observations of similarly structured, real galaxies.

\subsection{N-Body Systems}

We use three simulations in this study. The first, which we refer to here as simulation I, constitutes a barred galaxy with spiral structure. Originally presented in D06, where it is referred to as run L2.t12, it consists of a live disk immersed in a rigid halo. A complete description of the model parameters is contained in D06.

The second (spiral) simulation, which we refer to here as simulation II, is unpublished. It was designed with the main aim of generating strong spiral structure using the groove mode mechanism of Sellwood \& Lin (1989) and Sellwood \& Kahn (1991), in which dynamical instability develops from a "groove," or narrow feature, in the phase-space density at a particular angular momentum. A trailing spiral wave is generated, and at the Lindblad resonances of the wave, further grooves develop such that the 
TABLE 1

TWR Estimates for Simulation I

\begin{tabular}{|c|c|c|c|c|}
\hline $\begin{array}{c}\text { SA } \\
(\mathrm{deg})\end{array}$ & $\Omega_{b}$ & $\Omega_{s}$ & $r_{t}$ & $r_{c}$ \\
\hline $75 \ldots$ & 0.325 & 0.149 & 3.0 & 5.7 \\
\hline $45 \ldots \ldots$ & 0.327 & 0.111 & 2.4 & 4.8 \\
\hline $15 \ldots \ldots$ & 0.343 & 0.184 & 2.4 & 4.5 \\
\hline -15 & 0.269 & 0.172 & 2.7 & 4.8 \\
\hline$-45 \ldots \ldots \ldots \ldots \ldots \ldots \ldots \ldots$ & 0.313 & 0.199 & 3.0 & 5.4 \\
\hline$-75 \ldots \ldots \ldots \ldots \ldots \ldots \ldots \ldots$ & 0.303 & 0.203 & 2.4 & 6.0 \\
\hline Actual ........................ & 0.29 & 0.18 & 2.5 & 5.0 \\
\hline
\end{tabular}

NoTES.-TWR bar and spiral pattern speeds from the barred spira simulation listed here are estimated with TWR solutions calculated using a $\Delta r=0.3$ bin width for a range of SAs. The third and fourth columns list the connate estimates for $r_{t}$ and $r_{c}$. Values for the actual pattern speeds are shown in the last row.

instability is recurrent. Like simulation I, it consists of a rigid halo and live disk, but it also includes a live bulge component. The bulge constitutes $25 \%$ of the baryonic mass and is sufficiently concentrated that a bar is very slow in forming. The disk has Toomre $Q=1.2$; in order that a strong spiral was seeded, $\sim 6 \%$ of disk particles in a narrow angular momentum range were removed, leaving $4 \times 10^{6}-169,480$ particles including the bulge. The result, as can be seen in Figure 11 below, is the formation of a strong but transient spiral.

The last simulation in this paper, simulation III, is a double-barred galaxy generated using the method of Debattista \& Shen (2007). This high-resolution simulation consists of live disk and bulge components in a rigid halo potential. The model has $\sim 4.8$ million equal-mass particles, with $\sim 4$ million in the disk and $\sim 0.8$ million in the bulge such that the bulge has mass $M_{b}=0.2 M_{d}$, where $M_{d}$ is the disk mass. The initial Toomre $Q$ of the disk is $\simeq 2$. The formation of the secondary bar is induced by making the bulge rotate (to mimic a pseudobulge). More details of the simulation can be found in Shen \& Debattista (2007), where it is referred to as run $\mathrm{D}$.

As in D06, all lengths and velocities are here presented in natural units. We analyze a snapshot of each simulation at a single time step with the disk in the $(x, y)$-plane. By rotating the system about the $z$-axis, we assign a line-of-sight direction to establish the kinematical major axis. Another rotation about the $x$-axis gives the system an inclination $\alpha$ (chosen throughout at $\alpha=45^{\circ}$, unless otherwise specified). The snapshot is then projected onto the sky plane where $x_{\mathrm{obs}}=x$ and $y_{\mathrm{obs}}=y \cos \alpha$. For a given slice spacing, the slices along which the calculations occur are aligned perpendicular to the line-of-sight direction (parallel to the kinematical major axis). The orientation of these slices, which is identical to the disk PA in a real observation, is designated uniquely the slice angle (SA) in the studies that follow.

\subsection{Simulation I: Barred Spiral Galaxy}

The bar and spiral structure in this simulation, first presented in D06, is featured out to $r \sim 5.0$, clear in the surface density (Fig. 3) and its Fourier decomposition (Fig. 4). Beyond $r \sim 5.0$, the Fourier decomposition indicates the possible presence of a third pattern. With step models for $\Omega_{p}(r)$, then, we might reasonably extract pattern speeds for three distinct structures. However, the $m=2$ mode between $5.0<r<8.0$ is not associated with a strong surface density enhancement. And as remarked on in $\S 3.2$, Figure 19 in D06, reproduced here in Figure 5, shows that the pattern in this radial zone is maintained by multiple distinct pattern speeds. (Note that a real galaxy would not be disposed to

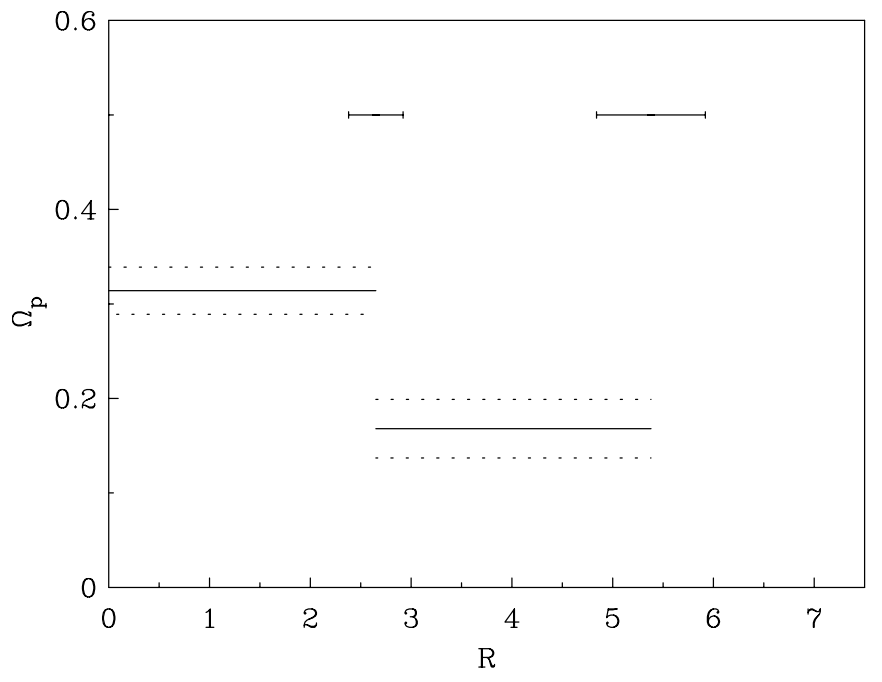

FIG. 6.-Best-fit solution and error bars from the TWR method applied to the barred spiral simulation averaged over six SAs. The bar $\Omega_{b}=0.31 \pm 0.02$ and spiral $\Omega_{s}=0.17 \pm 0.03$ are shown as solid lines with dashed errors. Errors in the bar-to-spiral transition $r_{t}=2.6 \pm 0.28$ and spiral termination radius $r_{c}=$ $5.38 \pm 0.54$ are represented by horizontal error bars at the top.

the analysis provided with this type of plot. It is available for this simulation, only, and we include it here for the sake of comparison.) In a clear account of regularization-induced bias, test solutions based on a three-pattern speed model have considerably larger $\chi^{2}$ than those parameterizing a bar and single spiral. We therefore reject models with a third pattern speed and use our cut scheme where solutions are generated without regularization up to a cut radius $r_{c}$, which parameterizes the end of the primary spiral pattern.

The actual pattern speeds of the bar and spiral structure to be reproduced by our solutions are $\Omega_{b}=0.29$ for the bar and a constant $\Omega_{s}=0.18$ for the spiral, as estimated from Figure 5 (from which we also estimate a bar-to-spiral pattern transition radius $r \sim 2.5$ ). In our models of $\Omega_{p}(r)$, we express the a priori assumption that the bar pattern speed is constant by using an $S$ like that in equation (14) but where, for $n>t, S$ is reflective of either a constant, linear, or quadratically varying spiral pattern speed. Solutions with spirals of order 0,1 , and 2 , then, have a total of 4,5 , and 6 degrees of freedom, respectively. The free parameter $t$ we restrict for all models such that $1.8<r_{t}<3.0$, according to bar length estimates from Figure $3\left(a_{B} \sim 2.2\right)$ and Figure 4.

For $\Delta r=0.3$ bins, we require a total of 71 slices (35 on each side) to reach the edge of the surface brightness at $r \sim 10.5$. This places the cut bin between $15<c<20$, according to the previously motivated restriction $4.5<r_{c}<6.0$ estimated from Figures 3 and 4 .

In light of the discussion in $\S 3.3$, we construct errors for our estimates to reflect the expected accuracy of TWR bar and spiral solutions given a particular observational scenario. Specifically, we perform the calculation for a range of SAs spanning the upper half-plane of the galaxy (quadrants I and II), namely, $\pm 15^{\circ}$, $\pm 45^{\circ}$, and $\pm 75^{\circ}$. Each SA corresponds to a unique disk PA. The resultant bar and spiral estimates generated using the $\Delta r=0.3$ bin width (to be discussed at length in the following sections) are listed in Table 1, and the average and rms of the best-fit solutions for this SA range are shown in Figure 6. There, horizontal error bars represent the dispersion in $r_{t}$ and $r_{c}$ in the solutions based on variations from SA to SA for the $\Delta r=0.3$ radial bin width.

Rewardingly, the best-fit solutions are quite accurate; the comprehensive spiral and bar estimates in Figure 6 are $6.7 \%$ and 

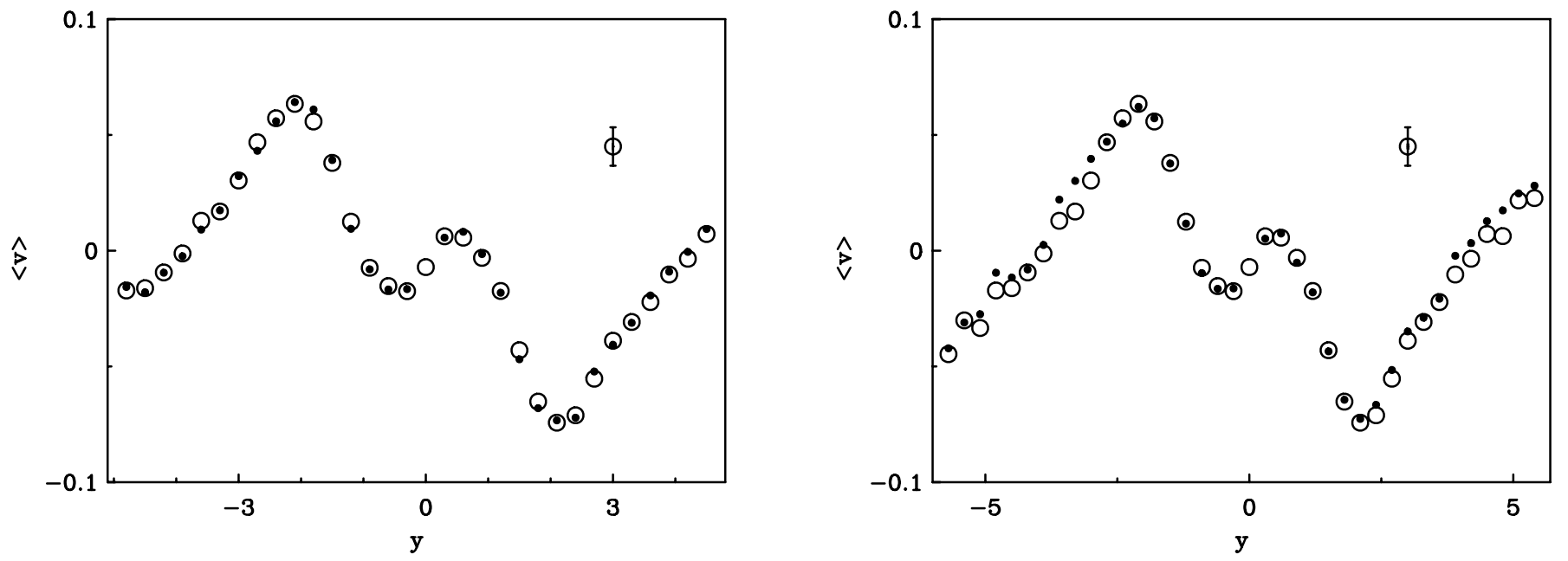

FIG. 7.-Comparison of model solution-reproduced ( filled circles) to actual (open circles) integrals $\langle v\rangle_{i}=b_{i} / \int \Sigma d x$ as a function of slice position $y$ for the barred spiral simulation for the best-fit constant $\Omega_{b}$, constant $\Omega_{s}$ solution with $r_{t}=2.4$ and $r_{c}=4.5$ (left) and constant $\Omega_{b}$, quadratic $\Omega_{s}$ solution with $r_{t}=2.7$ and $r_{c}=5.4$ for $\mathrm{SA}=15^{\circ}$ (right). Only those slices that show a contribution from bins inward of $r_{c}$ are shown. The adopted global error $\sigma^{\langle v\rangle}$ is shown in the upper right corner.

$8.3 \%$ from their actual values (with $\sim 8 \%$ error in $\Omega_{b}$ but a slightly larger error of $\sim 18 \%$ in $\Omega_{s}$ ). So, too, are the determinations of $r_{t}$ and $r_{c}$, according to Figures 4 and 5. Furthermore, our solutions correctly reproduce the functional form of the spiral pattern speed. At all SAs, out of all solutions with $r_{t}$ and $r_{c}$ within their restricted ranges, the lowest reduced $\chi^{2}$ solution corresponds to a constant spiral. Figure 7 shows a comparison between the actual and bestreproduced $\langle v\rangle$ at each slice position $y_{i}$ used in the calculation for the $15^{\circ} \mathrm{SA}$. The $\langle v\rangle$ reproduced by the best-fit constant bar and constant spiral solutions are shown in the left panel, while those from the optimum (lowest $\chi_{\nu}^{2}$ ) solution with a quadratic spiral are plotted in the right panel.

The close reproduction of the actual pattern speeds by the solutions in Figure 6 occasions further evaluation of strictly unregularized TWR calculations. Consider the values in radial bins inside $r \sim 2.0$ in typical unregularized solutions for this simulation (Fig. 2). That there is little to no indication of $\Omega_{b}=0.29$ in the left panel is perhaps not surprising: the large number of bins that accompany the choice of the small bin width would seem to guarantee a high level of noise propagated throughout the solution. However, in the slightly more stable solution with the wider bin width, the inner bins are still unrepresentative of the actual pattern speed in this zone. We can understand this as a systematic error introduced by the noise that not only propagates but also compounds as the full solution assembles from the outermost to the innermost radius; $\Omega_{p}(r)$ in a given bin reflects errors from all exterior radial bins, making the value in that bin more likely far removed from the actual value.

\subsubsection{Morphology-dependent Effects and Intrinsic Limitations}

The use of simulations that can be studied at multiple projections provides us with perhaps the most critical assessment for the accuracy of TWR solutions. Figure 6 suggests that the TWR method should perform well for any given viewing angle. However, although still quite small, the rms in each estimate is largely reflective of the nontrivial effect that the orientation of the pattern with respect to the SA used in the calculation can have on solutions.

We can understand the origin of the differences in solutions for the range of SAs in Table 1 by considering the impact of the limited azimuthal range of the bright spiral enhancement (clear from the surface brightness distribution in Fig. 3, where the spiral extends almost perpendicular to the bar major axis). That is, at all SAs the quadrature accumulates fragmentary information from the spiral since only some of the slices that cross the full radial zone of the spiral pattern intersect the strong spiral structure. But whereas both the bar and spiral estimates seem to suffer at SAs in quadrant II (i.e., positive SAs), our measurements of $\Omega_{s}$ in quadrant I are quite accurate. According to the morphology, in quadrant II it appears that the limited sampling of the spiral asymmetry implicit in slices other than those that also pass through the bar entails slightly less accurate spiral estimation.

To interpret the distinction between solutions from the two quadrants, consider the combined influence of regularization and our chosen weighting scheme. Specifically, since the regularizing $S$ induces the coupling of all bins within the same radial zone, even when the spiral-zone crossing slices do not intersect the strong spiral enhancement, the bins there inherit information from bins at the same radius from slices that do intersect the arms. According to our weighting scheme, however, this coupling is not uniform; the degree of support at each azimuth is influenced by the measurement errors for each slice, which grow larger from out to in. As a result, $\Omega_{s}$ is best constrained by information from slices passing through the outer radial zone of the spiral alone (not those passing through the bar). So when in quadrant II the spiral asymmetry appears in only the inner slices, $\Omega_{s}$ is less precise than when these outer slices clearly intersect the spiral arms. According to equation (8), since the bar estimate is directly related to that of the spiral, the result for these SAs is error in both $\Omega_{s}$ and $\Omega_{b}$.

The corresponding determination for the radial domain of the bar pattern, on the other hand, is not as obviously sensitive to this issue. Not only is the bar end reasonably well defined in both quadrants, but information from the bar that contributes to the parameterization of $r_{t}$ is reinforced with regularization in the manner described above. The spiral termination radius, too, seems fairly consistent from SA to SA. But since the asymmetry is weaker (and there is less information) at that location in the disk, we find that this parameter requires the most restriction (indeed, our determinations of $r_{c}$ completely span the allowed range).

Overall, then, our determinations for $r_{t}$ and $r_{c}$ are stable and accurate, each with less than $11 \%$ error, even in quadrant II. Nevertheless, since the pattern speed estimates from SAs in quadrant I seem to comparatively benefit from the high quality of information 


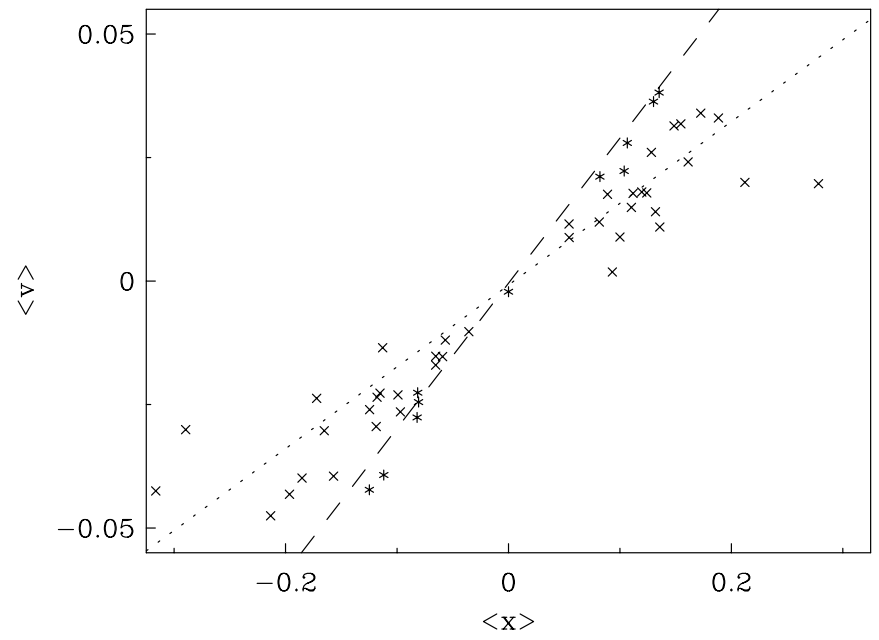

FIG. 8.-Plot of $\langle v\rangle$ vs. $\langle x\rangle$ for all slices with $|y|<5.1$ for the barred spiral simulation at $\mathrm{SA}=-45^{\circ}$. The dashed line is the best-fit straight line to the inner 11 slices (asterisks), while the dotted line is the best-fit straight line to all slices shown (asterisks and crosses).

from both patterns, we conclude that position angles that provide the most uniform slice coverage of all patterns in the disk are preferred.

The largest disparity between step model solutions from various SAs can largely be attributed to limitations in determining the location of the transition between the two patterns. That is, solutions are affected by the finite bin width inherent to the numerical calculation; slight incompatibility between the actual transition and that to which the solution is limited (given the bin width) can result in errors around $10 \%$. This is a more pervasive effect than morphology alone and more obvious with the use of a slightly larger bin width than $\Delta r=0.3$. Nonetheless, we can make several informed inferences about the result of the finite bin width by considering the nature of the TWR calculation. Specifically, since the transition determines the contributions of inner and outer patterns to the integral on the right-hand side of equations (4) and (7) through the matrix elements $K_{i j}$, a mismatch between the transition bin and the actual transition radius will corrupt the separation of the contributions of the two patterns. Note that this is precisely the source of the covariance between model parameters intimated in $\S 3.3$.

To understand the effects of (minor) radial pattern misassignment, consider a step function solution that parameterizes the extent of a bar pattern speed along with that of a spiral. When the transition bin between the two patterns underestimates the actual transition by a fraction of the bin width, for instance, we would expect the numerical calculation to effectively subtract off from $b_{i}$ a contribution from the (lower) outer pattern where a higher pattern actually exists (e.g., eq. [8]), slightly raising the value of the inner pattern speed. Conversely, we expect an overestimation of the transition to result in a slightly lower inner pattern speed. More subtle effects can occur, however, depending on the geometry of the patterns, as illustrated for this simulation in $\S$ 4.2.4.

For a range of SAs, we emphasize that the resultant errors, in possible combination with an undersampled transition, are minimal, as long as the bin size is sufficiently small, and are not the result of vastly different transitions for each SA; we find that the transition between patterns in the best solutions is generally relatively stable with changes in SA. This may be unexpected from the perspective of the traditional TW method since the projected length presented to the slices by the bar will depend on the relative
TABLE 2

Optimal TW Bar Estimates for Simulation I

\begin{tabular}{|c|c|c|c|}
\hline $\begin{array}{c}\text { SA } \\
(\mathrm{deg})\end{array}$ & $N$ slices & $\Omega_{p}$ & $\sigma$ \\
\hline $75 \ldots$ & 7 & 0.270 & \pm 0.010 \\
\hline $45 \ldots \ldots \ldots \ldots \ldots \ldots \ldots$ & 11 & 0.282 & \pm 0.062 \\
\hline $15 \ldots$ & 17 & 0.208 & \pm 0.007 \\
\hline-15 & 15 & 0.248 & \pm 0.026 \\
\hline$-45 \ldots \ldots \ldots$ & 11 & 0.294 & \pm 0.008 \\
\hline$-75 \ldots \ldots \ldots$ & 9 & 0.233 & \pm 0.053 \\
\hline
\end{tabular}

Notes.-All entries originate through the use of an optimal number of slices spaced at $\Delta y=0.3$. The number of slices $N$ used in the TW calculation is indicated.

orientation of the slices with its major axis (and slice orientation errors tend to be large, as detailed next for this simulation).

$$
\text { 4.2.2. } T W R \text { versus } T W
$$

In light of the above results, we next examine the improvements available to pattern speed estimation using the TWR calculation relative to the TW method. We specifically compare the bar pattern speed estimates arrived at using the TWR method with those using fully extended TW integrals. Although TW estimates of $\Omega_{b}$ in the presence of a secondary structure may also be attempted using truncated integrals that extend to just past the end of the bar (such that information from the bar alone is dominant), we examine the former case for two reasons: (1) to compare the two methods under identical conditions (i.e., using the same data points $\langle v\rangle_{i}$ ) and (2) to study the influence of the relatively weak spiral (and evaluate the assumption of negligible nonaxisymmetric motions beyond the bar).

Although we use fully extended integrals to perform the TW calculation, even when making estimates of the bar pattern speed, the innermost slices clearly supply evidence for a bar pattern speed that is distinct from that of the other structure in the disk. Figure 8 shows a typical plot of $\langle v\rangle$ versus $\langle x\rangle$ for this simulation, where the inner 11 slices are indeed best fitted by a steeper slope than for all slices.

For all of the other SAs studied in the previous section, we measure $\Omega_{p}$ with a unique number of bar-crossing slices. This is intended to reproduce an optimal TW observing strategy that makes use of only those slices that intersect the enhanced bar surface density. That is, since the projected length presented to the slices by the strong bar structure depends on the relative orientation of slices with the major axis of the bar, the number of slices that intersect the bar enhancement varies from SA to SA (from 7 to 17 for this simulation at the six studied SAs). Table 2 lists this optimal number of slices $N$ at each SA along with the corresponding pattern speed estimate. All entries in the table correspond to slopes of best-fit straight lines (and the corresponding intrinsic scatter) in plots of $\langle v\rangle$ versus $\langle x\rangle$.

On inspection, Table 2 seems to suggest that, even in the presence of the spiral asymmetry, information from the bar is maximal in the bar-crossing TW integrals. Despite also reflecting a contribution from the spiral pattern, these bar estimates are fairly accurate (although presumably not as accurate as would be the case for a strictly SB0 galaxy such as NGC 7079). However, as in Table 3, if we extend the slice coverage at each SA out to $|y| \sim$ 2.4 using the inner 17 slices (at $\Delta y=0.3$ spacing; closer to the full extent of the radial zone of the bar pattern, according to Fig. 6), then the quality of the TW estimates diminishes with the inner, bar pattern speed estimates approaching that from a fit to all slices 
TABLE 3

Traditional TW Estimates for Simulation I

\begin{tabular}{|c|c|c|c|c|}
\hline \multirow{2}{*}{$\begin{array}{c}\text { SA } \\
\text { (deg) }\end{array}$} & \multicolumn{2}{|c|}{ INNER 17 SLICES } & \multicolumn{2}{|c|}{ All Slices } \\
\hline & $\Omega_{p}$ & $\sigma$ & $\Omega_{p}$ & $\sigma$ \\
\hline $75 \ldots$ & 0.234 & \pm 0.004 & 0.115 & \pm 0.008 \\
\hline $45 \ldots \ldots$ & 0.217 & \pm 0.045 & 0.094 & \pm 0.018 \\
\hline $15 \ldots \ldots \ldots$ & 0.208 & \pm 0.007 & 0.157 & \pm 0.009 \\
\hline .................. & 0.216 & \pm 0.016 & 0.121 & \pm 0.007 \\
\hline-45 ........................ & 0.278 & \pm 0.009 & 0.166 & \pm 0.009 \\
\hline$-75 \ldots \ldots \ldots \ldots \ldots \ldots \ldots \ldots \ldots \ldots \ldots \ldots \ldots \ldots \ldots$ & 0.175 & \pm 0.010 & 0.128 & \pm 0.010 \\
\hline
\end{tabular}

Notes.-As in Table 2, the TW estimates for this SA range are generated with slices spaced at $\Delta y=0.3$. Here the second and third columns list the estimates $\Omega_{p}$ along with errors $\sigma$ using the inner 17 slices, respectively, while the fourth and fifth columns are from a fit to all slices with $|y| \leq 5.0$, out to the inferred spiral end.

with $|y|<5.1$; even the true bar-crossing slices contain nonnegligible information from the spiral pattern.

Unlike the TW method, the TWR method is not relegated to the use of only those slices where the bar contribution is maximized. In principle, the inner solutions are accessible through the very use of information from beyond the bar (namely, from the zone of the spiral) and are improved when this information is radially coupled (e.g., by regularization). This aspect of the calculation allows for the return of pattern speed solutions without reference to an assumed pattern extent and provides, moreover, an independent means of determining the radial domain of patterns.

Of course, this is not to imply that a deficiency of usable slices in TW estimates prevents accurate pattern speed measurement, or determination of pattern extent, for that matter; using slices that cross primarily through the enhanced emission from the bar, TW estimates from SB0 galaxies have been successfully used to observationally confirm that bars end at or inside their corotation radii. However, it does suggest that in the presence of nonnegligible asymmetry exterior to the bar, TW bar estimates are susceptible to errors introduced by the use of slices positioned near the bar end, which are presumed to reflect the bar pattern speed but in reality include a significant contribution from this structure. Consider a typical plot of $\langle v\rangle$ versus $\langle x\rangle$ for bar-crossing slices. The best-fit slope determination for $\Omega_{p}$ from such a plot is primarily governed by slices with the largest $\langle v\rangle$ and $\langle x\rangle$. Since in SB0 galaxies $\langle v\rangle$ and $\langle x\rangle$ approach zero in slices at or near the projected bar end (since they are presumably too far past the strong bar structure to contain information about the bar and mark, rather, a return to axisymmetry in the disk), even when TW estimates consider these "near zero" slices, they contribute minimally to estimates for $\Omega_{p}$. (See Debattista [2003], where studies with a simulated SB0 include a number of slices sampling the full extent of the bar.) But when there is considerable asymmetric structure present beyond the end of the bar, similarly positioned slices will reflect this contribution, impairing measurement of the true bar pattern speed.

In the barred spiral simulation, this consequence can be characterized on inspection of $\langle x\rangle,\langle v\rangle$, and $\Omega_{p}^{\mathrm{TW}}=\langle v\rangle /\langle x\rangle$ as a function of the limit of integration $X_{\max }=X_{0}$ in a typical slice at $y=1.2$ (see Fig. 9 for $\mathrm{SA}=-75^{\circ}$ ). $\Omega_{p}^{\mathrm{TW}}$ for this slice, which, according to our estimate for $a_{B}$, crosses the outer region of the bar, actually seems more reflective of the spiral pattern speed (beyond the discontinuity at $X_{0} \sim 3.2$ ). Indeed, past $X_{0} \sim 3.2$ where $\langle x\rangle$ crosses zero we can infer that this bar-crossing slice contains substantial participation from the spiral; both $\langle x\rangle$ and $\langle v\rangle$ decrease to a dip between $X_{0}=4.0$ and 6.0 before reaching a plateau. This is evi-

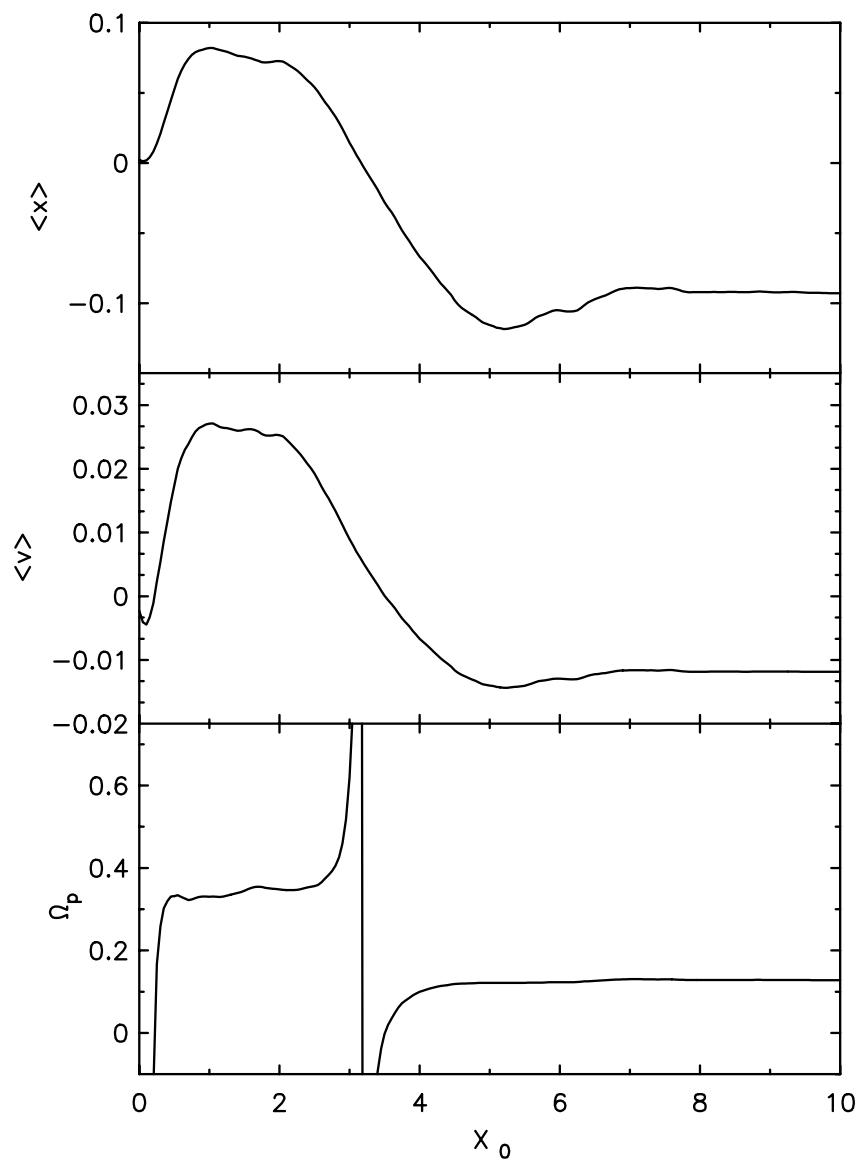

FIG. 9.- Variation of $\langle x\rangle$ (top),$\langle v\rangle$ (middle), and $\Omega_{p}^{\mathrm{TW}}=\langle v\rangle /\langle x\rangle$ (bottom) with $X_{0}$ for the slice at $y=1.2$ with $\mathrm{SA}=15^{\circ}$ for the barred spiral simulation.

dence that, despite the relative weakness and limited extent of the spiral as indicated by the surface brightness distribution, the asymmetry in the disk should not be considered dominated by the bar alone. Moreover, this is a clear indication that the TWR calculation, which identifies and effectively removes the spiral contribution from the fully extended $\Sigma$-weighted velocity integrals, can improve on traditional TW bar estimates that also use fully extended integrals. Of course, as displayed by Figure 9, integrating only between $\pm X_{0} \sim 2.5$ may alone provide a reasonable bar pattern speed and relieve all other TW slices from the spiral contribution. However, this would provide only a single pattern speed estimate where two are possible; using the TWR method and fully extended integrals, both $\Omega_{b}$ and $\Omega_{s}$ can be measured.

Figure 9 also raises a crucial point related to the required limit of integration along each slice in the TWR calculation: if there is a clear plateau in the integrals reached before the edge of the map boundary, why not simply truncate the integrals where they have converged (common to TW estimates) rather than use integrals extending to the edge of the surface brightness and that present outermost bins that we demand must be cut (that is, calculated without regularization), anyway? The plateau reached at $X_{0} \sim$ 8.0 would suggest that truncating the integral there could suitably account for information from the major sources of asymmetry in the disk. However, this same distinction is not clearly shared by all slices, especially those at large $|y|$. If we associate the plateau in this slice at $y=1.2$ with the limit of integration $X_{\max }=8.2$ and hence the total radial extent $R_{\max }$ required of the quadrature where $R_{\max }=\left[X_{\max }^{2}+(y / \cos \alpha)^{2}\right]^{1 / 2}$, then this locates the outermost slice position at $y_{\max }=\left(R_{\max }-\Delta r\right) / \cos \alpha$, as well 
TABLE 4

PA Errors in TW and TWR Estimates for Simulation I

\begin{tabular}{|c|c|c|c|c|}
\hline \multirow[b]{2}{*}{ МЕтHоD } & \multicolumn{2}{|c|}{$\delta_{\mathrm{PA}}=+2^{\circ}$} & \multicolumn{2}{|c|}{$\delta_{\mathrm{PA}}=-2^{\circ}$} \\
\hline & $\Omega_{b}$ & $\Omega_{s}$ & $\Omega_{b}$ & $\Omega_{s}$ \\
\hline \multirow{2}{*}{$\mathrm{TW}^{*}$} & 0.179 & 0.162 & 0.354 & 0.115 \\
\hline & \pm 0.055 & \pm 0.058 & \pm 0.04 & \pm 0.02 \\
\hline \multirow[t]{2}{*}{ 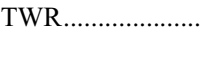 } & 0.214 & 0.211 & 0.380 & 0.158 \\
\hline & \pm 0.031 & \pm 0.045 & \pm 0.042 & \pm 0.023 \\
\hline
\end{tabular}

NoTES.-Entries correspond to average bar and spiral estimates from SAs in quadrant I $\left(-15^{\circ},-45^{\circ}\right.$, and $\left.-75^{\circ}\right)$ with PA errors $\delta_{\mathrm{PA}}=+2^{\circ}$ and $-2^{\circ}$. Estimates from both the traditional TW (asterisk indicates bar estimates using the nominal number of slices for each SA listed in Table 2) and TWR methods are listed.

as the extent of the integral along this slice. It is easy to check that (at least for this slice orientation) the integral has not achieved convergence by this point, nor have most other integrals in the disk by $R_{\max }$.

Rather than risk ignoring information critical for characterizing the patterns uniformly throughout the disk, then, we choose to include in the quadrature all information out to the edge of the surface density. Indeed, this serves to perform a function similar to truncating TW integrals. The difference in the two procedures arises from the fact that while the limits of integration for each individual slice can be adjusted for a given structure of interest in the TW calculation, the quadrature in the TWR method delineates specific bounds that must encompass complete information from all extended patterns in the disk.

\subsubsection{Systematic Errors}

In this section we use the barred spiral simulation to consider the errors introduced to TWR pattern speed estimates in real galaxies. We can expect errors in the assumed $\mathrm{PA}_{\text {disk }}$ to dominate errors in the TWR calculation, given that such errors translate significantly to inaccuracies in the traditional TW calculation via the line-of-sight velocity integral, which is also, of course, a prominent feature of the radial TW equation. Table 4 summarizes the results for a standard PA error of $\delta_{\mathrm{PA}}= \pm 2^{\circ}$ on the SAs in quadrant I chosen for their advantages, as evidenced by the discussion in $\S 4.2 .1$. The average and $\mathrm{rms}$ for bar and spiral estimates from both the TWR and the traditional TW methods are listed. (The TW "bar" estimates are obtained by fitting to the inner, nominal number of slices listed in Table 2, while all slices with $|y|<5.1$ are considered in the "spiral" estimates.)

Even this small $\delta_{\mathrm{PA}}$ introduces considerable errors (relative to the known pattern speeds) to both types of bar estimates. These errors in $\Omega_{b}$ can be many times larger than the formal rms. But whereas the errors are comparable in the TWR and TW bar estimates, the errors in the spiral estimates tend to be smaller with TWR than TW. Rewardingly, with this error not only are the TWR spiral solutions still definitively constant and accurate to $\sim 15 \%$, but the radial domains of both pattern speeds are still well determined. The transition between the bar and spiral $r_{t}$ and the termination radius of the strong spiral pattern $r_{c}$ are effectively unchanged from the $\delta_{\mathrm{PA}}=0$ case; for both $\delta_{\mathrm{PA}}=+2^{\circ}$ and $\delta_{\mathrm{PA}}=-2^{\circ}$ we find $r_{t}=2.8 \pm 0.37$ and $r_{c}=5.4 \pm 0.46$.

Besides the effects on $\mathrm{PA}_{\text {disk }}$ measurement as studied by Debattista (2003), galaxy inclination and ellipticity play perhaps more prominent roles as sources of error in TWR solutions relative to traditional TW estimates. Presumably, large inclination errors will prevent the association of information into accurate radial bins, given that $r=y_{\mathrm{obs}} / \cos \alpha$. We expect this effect to be

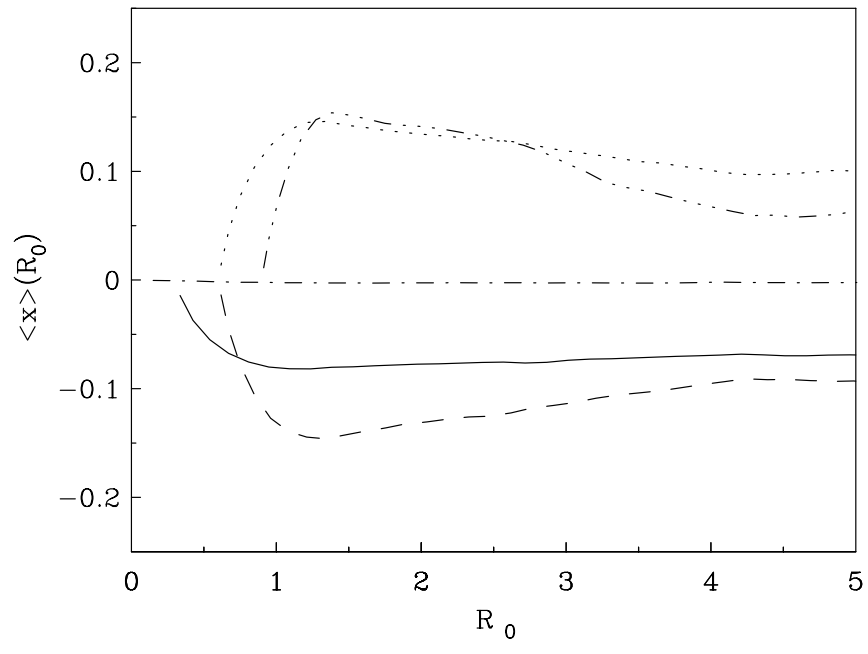

FIG. 10.-Variation of $\langle x\rangle=\int_{-X_{0}}^{X_{0}} \Sigma x d x / \int_{-X_{0}}^{X_{0}} \Sigma d x$ with $R_{0}=\left(y_{i}^{2}+X_{0}^{2}\right)^{1 / 2}$ for five slices at $\mathrm{SA}=45^{\circ}$ (dot-dashed line: $y_{1}=0.0$; dashed line: $y_{2}=-0.6$; solid line: $y_{3}=-0.3$; dotted line: $y_{4}=0.6$; triple-dot-dashed line: $\left.y_{5}=0.9\right)$ in the barred spiral simulation. The plateau range indicates a transition between $R_{0} \sim 1.0$ and 1.5 .

minimal at moderate inclinations since $d r \propto d \alpha \sin \alpha$, and most significant at small inclinations where one would generally find that the difficulty in inferring in-plane morphology and kinematics makes the TW method impractical in any case. At a moderate, $45^{\circ}$ inclination we find that the barred spiral solutions for $\pm 3^{\circ}$ inclination error differ from the actual pattern speeds by only the change in $\sin \alpha$ introduced by the line-of-sight velocity.

\subsubsection{Transition Misidentification}

We have shown that the regularized TWR method can be used to parameterize the number and radial domain of multiple pattern speeds in a single disk. Formally, the contribution of each to the line-of-sight velocity integral is established through the designation of a transition between patterns. In our scheme this transition is a free parameter, but the method, of course, could plausibly assimilate other transition identification methods to similar effect, much like that in the Maciejewski (2006) adaptation of the TW method. Specifically, in Maciejewski (2006) a plateau in the integrals $\int_{-X_{0}}^{X_{0}} \Sigma x d x$ with variation in $X_{0}$ is associated with the transition from an inner to an outer pattern (for details see Maciejewski 2006). This transition is then used to separate the disk surface brightness into two unique components (one for an inner secondary bar, one for an outer primary bar), thereby governing the decoupling of the pattern speeds.

We here pursue this type of diagnostic for the case of the simulated barred spiral in order to test the reliability of employing the TWR method with such independent evidence for pattern extent. Figure 10 plots the values of $\int_{-X_{0}}^{X_{0}} \Sigma x d x / \int_{-X_{0}}^{X_{0}} \Sigma d x$ as a function of $R_{0}$ for five bar-crossing slices $\left(y_{1}=0.0, y_{2}=-0.6, y_{3}=\right.$ $\left.-0.3, y_{4}=0.6, y_{5}=0.9\right)$ at $\mathrm{SA}=45^{\circ}$, where $R_{0}=\left(y_{i}^{2}+X_{0}^{2}\right)^{1 / 2}$.

For this projection, there seems to be a plateau at $R_{0} \sim 1$. . We note that this value is smaller than the bar pattern extent indicated by our best-fit solutions (and the major-axis bar length estimated by inspection of the surface brightness distribution) and, furthermore, the same analysis performed over the range of SAs does not always as clearly show the same behavior. Presumably, this particular value is more indicative of the bar minor-axis length than the full radial zone of the bar pattern, since slices at a $45^{\circ} \mathrm{SA}$ sample along $X$ perpendicular to the bar major axis; other slice orientations are similarly limited to sampling the bar according to its specific projection. 
TABLE 5

TWR Estimates for Simulation I with a Misidentified Transition

\begin{tabular}{|c|c|c|c|c|}
\hline $\begin{array}{c}\text { SA } \\
(\mathrm{deg})\end{array}$ & $\Omega_{b}$ & $\Omega_{s}$ & $r_{t}$ & $r_{c}$ \\
\hline $75 \ldots$ & 0.376 & 0.193 & 0.9 & 5.7 \\
\hline 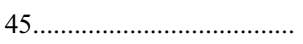 & 0.348 & 0.152 & 0.9 & 4.8 \\
\hline $15 \ldots \ldots \ldots$ & 0.367 & 0.191 & 1.2 & 4.5 \\
\hline-15 & 0.336 & 0.186 & 1.2 & 4.5 \\
\hline-45 & 0.336 & 0.212 & 1.2 & 5.7 \\
\hline-75 & 0.313 & 0.200 & 1.2 & 5.7 \\
\hline
\end{tabular}

NOTES.-All bar and spiral pattern speeds listed here are estimated from TWR solutions where $r_{t}$ is restricted to between 0.9 and 1.5. As in Table 1 , solutions are calculated using a $\Delta r=0.3$ bin width. The fourth and fifth columns list the connate estimates for $r_{t}$ and $r_{c}$.

Allowing that information from the full radial zone of the bar is not manifest in this type of indicator, we here proceed to assess the consequences for inner and outer pattern speed estimates when the transition between the two patterns is misidentified. Table 5 lists the TWR bar and spiral estimates for each of the SAs studied in the previous sections, where we have limited the transition to $0.9<r_{t}<1.5$. At all SAs, this error of several bins in $r_{t}$ causes an overestimation of the inner pattern speed. The outer pattern speed, on the other hand, although slightly raised, is still reassuringly accurate. We can interpret the inaccuracy in $\Omega_{b}$, then, as the result of the misassociation of information from one pattern to the other via equation (8), as discussed at the end of $\S 4.2 .1$. In addition, we can attribute the greater inaccuracy in solutions from quadrant II to the reasons discussed in $\S 4.2 .1$. Although the subtleties in Table 5 are most likely specific to this simulation, we emphasize that systematic pattern speed errors introduced by transition misidentification are generic to the nature of the calculation.

One of the greatest strengths of the TWR calculation is that the transition is in principle a free parameter (within limits) and need not be restricted to a single, predetermined value. We therefore recommend letting the results of the TWR calculation speak for themselves: given sufficient resolution and reasonable measurement errors, step model solutions with the most realistic transition should be recognizable by how well they reproduce the actual $\langle v\rangle$. Since the transition determines the separation of the patterns by interpreting the contribution made to these integrals by each, the natural result is the most accurate determination of the pattern speeds possible.

\subsection{Simulation II: Spiral Galaxy}

In the previous section we showed that the TWR method is capable of detecting and measuring a constant spiral pattern speed that spans less than one-third of the disk. Here we test the aptitude of the TWR method in measuring a radially varying spiral pattern speed that subsists over a large radial zone. Since the strong spiral surface density enhancement in this simulation (Fig. 11) has only moderate azimuthal range like the spiral in $\S 4.2$, we further explore the likely limitations intrinsic to detecting spiral nonaxisymmetry with a given slice orientation.

The two-armed spiral featured in this simulation extends over a large portion of the disk and is strong both in the surface brightness distribution (Fig. 11) and as traced by departures from axisymmetric rotation (streaming motions) in the velocity field (Fig. 12). We estimate the extent of the spiral structure from that of the dominant $m=2$ component in the Fourier power spectrum plotted in Figure 13. With the expectation, then, that the

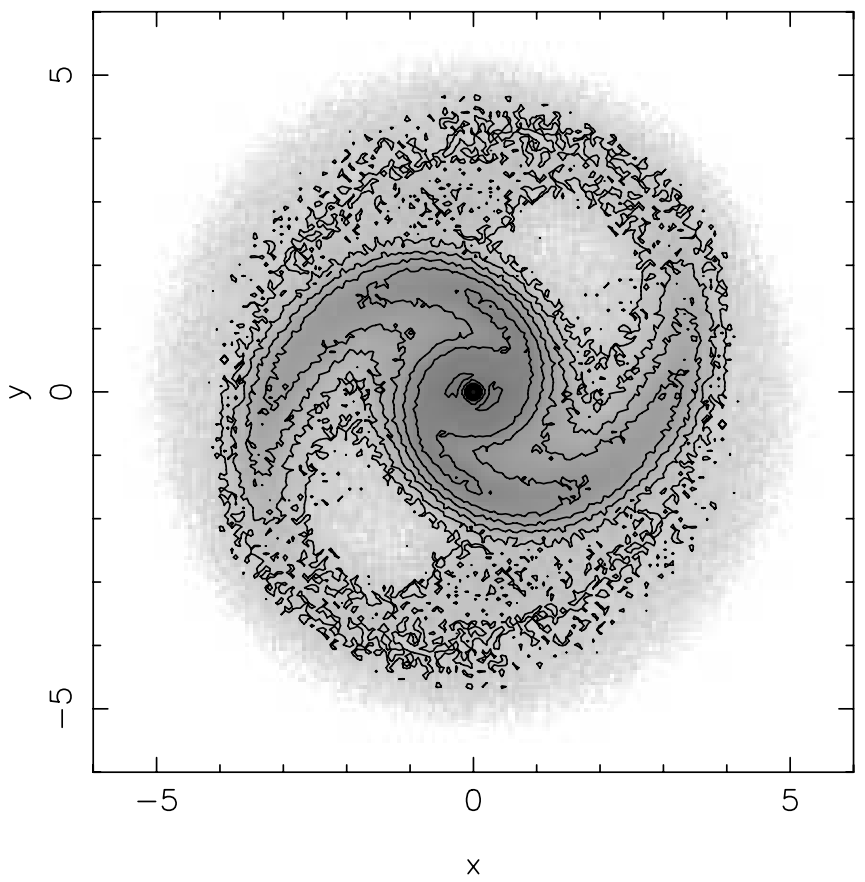

FIG. 11.-Face-on display of the spiral simulation's surface brightness distribution projected with $\mathrm{a}-30^{\circ}$ rotation about the $z$-axis, with orientation as in Fig. 3.

spiral structure exists between $0.5 \lesssim r \lesssim 3.5$, we restrict our spiral pattern speed solutions to the radial zone $r_{t}<r<r_{c}$ bordered at the innermost and outermost radii by two independent sets of unregularized bins. (For our models, $0<r_{t}<1.0$ and $2.8<r_{c}<4.2$.) Between the radii marked by the free (although restricted) parameters $r_{t}$ and $r_{c}$, we allow each spiral pattern speed solution to vary with radius as an $n$ th-order polynomial where $n=0-2$.

The two sections of "place-holding" unregularized bins serve to isolate the solution in the radial range of interest; by minimizing errors due to either incorrectly incorporating or imposing an ill-prescribed form to bins outside the radial zone of the spiral,

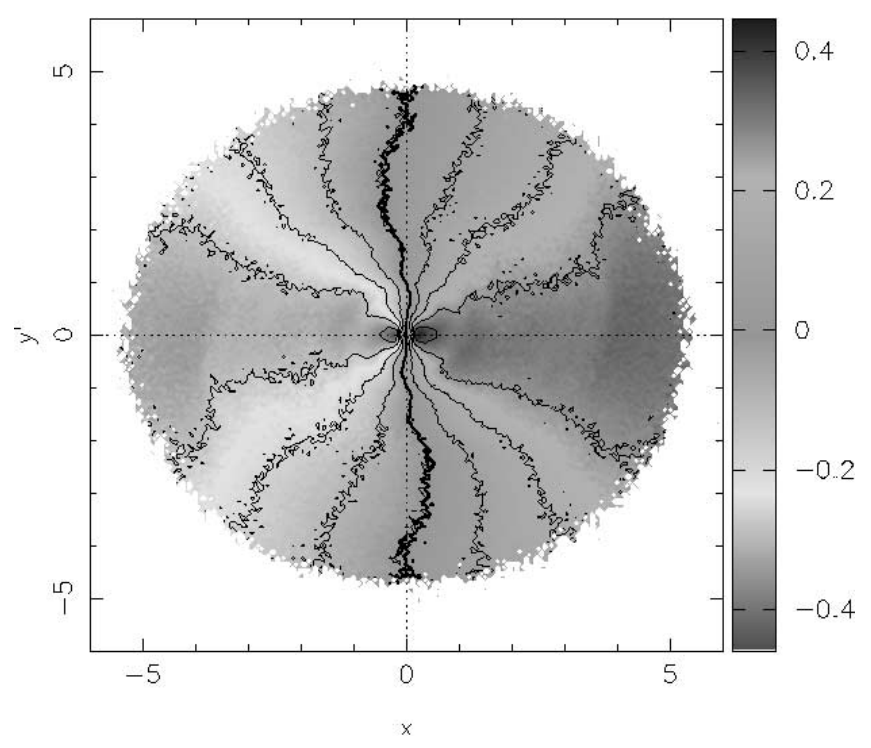

FIG. 12.-Face-on display of the spiral simulation's velocity field projected with no rotation about the $z$-axis shown here with the kinematical major axis running from left to right. Contours are spaced at $\Delta v=0.1$. 


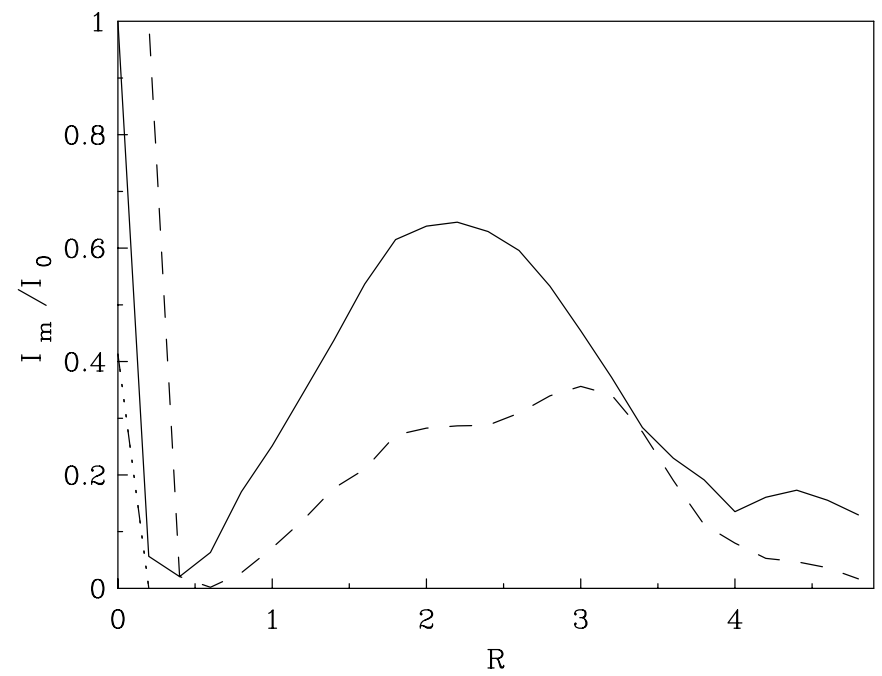

FIG. 13.-Fourier power spectrum of the spiral simulation's surface brightness distribution shown in Fig. 11. Modes up to $m=4$ are plotted as a function of radius: $m=1$ (dotted line), $m=2$ (solid line), $m=3$ (triple-dot-dashed line), and $m=4$ (dashed line).

we reduce the introduction of inaccuracies to the spiral pattern speed solution. The first, outermost section covers the radial zone identified by visual inspection of the surface brightness (and substantiated by the Fourier power spectrum) where the dominant spiral ceases to extend and where the weakness of the Fourier components suggests a region without a noticeable pattern. As a result, we consider this zone unsuitable for reliable extraction of a pattern speed. (This last point can also be evidenced by traditional TW values from slices that pass solely through the outermost disk; the low counts there lead to a large degree of variation in $\Omega_{p}$ estimates from slice to slice). The second, innermost section covers the radial zone inside $r_{t} \sim 1.0$. Within this radius we do not expect to be able to extract a realistic pattern speed solution since neither the morphology, velocity field, nor the power spectrum indicates a departure from axisymmetry.

Lacking such evidence in the innermost radii, it would be entirely possible to proceed without the use of unregularized inner bins (as would be the case when the indicators for such a measure are perhaps less obvious). Indeed, allowing an independent regularized solution to exist at $r<r_{t}$, the TWR calculation still performs well; since these bins cover a rather small region of the disk and thereby contribute minimally to the $\chi^{2}$ through only the innermost slices, we find that the spiral solution outside of this region closely resembles that in the case where the inner bins are unregularized. However, we proceed in the manner described above with the expectation that, if only minorly, our spiral pattern speed solution will be improved.

The actual pattern speed at the time of the snapshot shown in Figure 11 as derived from the time evolution of the phase of the $m=2$ component is plotted as a function of radius in Figure 14. This plot confirms that within $r \sim 1.0$, the pattern speed is ill quantified, with the values for $\Omega_{p}$ at the innermost radii oscillating between positive and negative values outside of the vertical range of the plot. At the largest radii, the pattern speed is characterized by scatter presumably reflective of the lack of a noticeable pattern in Figure 11.

The pattern speed between $1.0 \lesssim r \lesssim 3.0$ to be reproduced by our solutions shows high-order variation with radius. Inside of $r \sim 2.0$ where the pattern speed is at a maximum $\left(\Omega_{p, \max } \sim 0.3\right)$, the pattern seems to be unwinding, while at larger radii the pattern speed decreases with increasing radius.

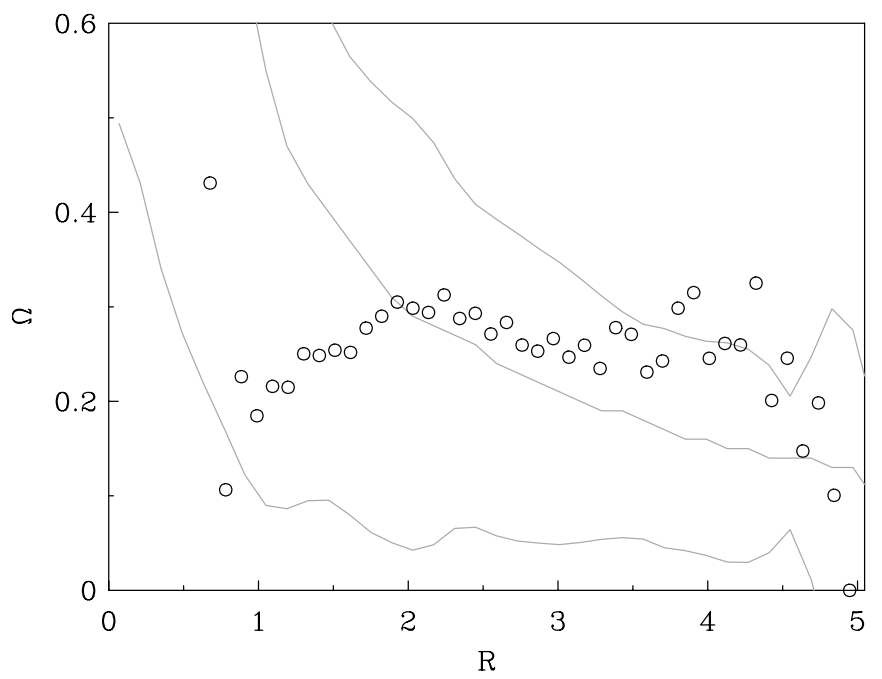

FIG. 14.-Plot of $\Omega_{p}$ as a function of radius for the spiral simulation as derived from the time evolution of the $m=2$ component. Curves for $\Omega$ and $\Omega \pm$ $\kappa / 2$ are shown in gray.

This behavior is only modestly indicated by traditional TW estimates. In plots of $\langle v\rangle$ versus $\langle x\rangle$ (see Fig. 15 for a comparison of plots generated at four SAs), adjacent slices trace out a figureof-eight shape characteristic of complex radial behavior (namely, winding). However, the evidence for a variable best-fit slope (expected for a pattern speed that unwinds and winds) is not comprehensive or even readily apparent in all cases. (In fact, all slices are seemingly well fitted with a single slope.) The radial dependence of $\Omega_{p}$ becomes more apparent on inspection of the variation of $\Omega_{p}$ with slice position (see Fig. 16), but again, the radial dependence gets smoothed out since $\Omega_{p}$ for each slice is the result of averaging over all sampled radii. Furthermore, for the four SAs shown in Figure 16, there is no single radial behavior implied by all.

The TWR solutions, on the other hand, are capable of clearly displaying high-order variation in $\Omega_{p}(r)$. The average and rms of the best-fit solutions with $\Delta r=0.2$ bins at six different SAs $\left( \pm 15^{\circ}, \pm 45^{\circ}\right.$, and $\left.\pm 75^{\circ}\right)$ are plotted in Figure 17 between the average values of $r_{t}$ and $r_{c}$. For each SA, we find that the best-fit solution is quadratically varying, correctly reproducing the winding behavior present in the actual pattern speed. However, the parameters $r_{t}, r_{c}$, and the size and location of $\Omega_{p, \max }$ differ slightly in each solution. These differences tend to reflect the influence of morphology and slice orientation, as in $\S 4.2 .1$. The value of the peak in $\Omega_{p}(r)$, for example, is higher and more pronounced in solutions from quadrant II than quadrant I; at positive (negative) SAs $\Omega_{p, \max } \sim 0.34(0.27)$, on average. Together with differences in the location where $d \Omega_{p}(r) / d r=0$ (which varies for the six SAs within the range $1.6<r<2.2$ ), on averaging, the result is a slightly undermeasured peak value occurring at $r \sim 1.8$ (in accord with the actual location).

The vertical error bars on bins at large and small $r$ similarly reflect variations in the location of $r_{c}$ and $r_{t}$ with SA. Principally, the solution suffers contamination from bins that perhaps yet contain information from the inner or outer axisymmetric zones. But we also find that solutions in quadrant II tend to decrease from $\Omega_{p \text {, max }}$ to a value $r_{c}$ that is further in by about $10 \%$ than for solutions using slices at the perpendicular orientation. Nevertheless, we find the average $r_{t}=0.8 \pm 0.2$ and $r_{c}=3.2 \pm 0.3$ to be in agreement with the bounds of the spiral pattern indicated by the disk surface density and its Fourier decomposition. 

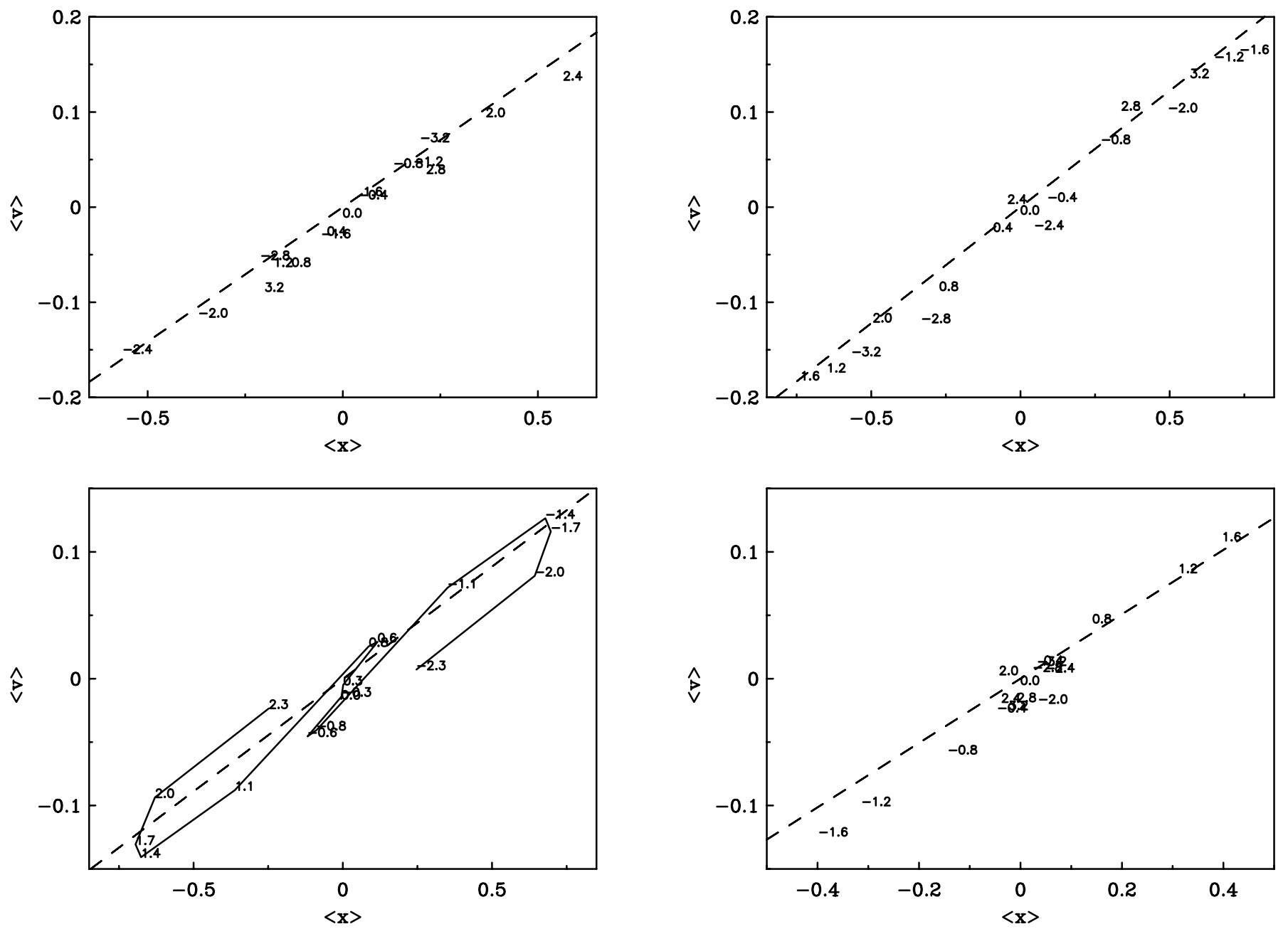

FIG. 15.-Plot of $\langle v\rangle$ vs. $\langle x\rangle$ for every other slice spaced at $\Delta y=0.4$ for the spiral simulation at four SAs (clockwise from top left: $45^{\circ}, 15^{\circ},-15^{\circ}$, and $-45^{\circ}$ ). Each slice is labeled by its distance from the galaxy major axis $y_{i}$. Adjacent apertures in the $-45^{\circ}$ case are connected by a solid line; this SA shows the clearest signature of winding. The dashed line in all plots is the best-fit straight line to all apertures shown.

Despite these PA-dependent effects, the values implied by the average solution $\left(\Omega_{p, \max },\left.\Omega_{p, \min }\right|_{r=0.8}\right.$, and $\left.\left.\Omega_{p, \min }\right|_{r=3.2}\right)$ are accurate to within $5 \%, 11 \%$, and $10 \%$, respectively. That we have correctly reproduced the high-order variation of $\Omega_{p}(r)$, regardless of SA, however, is perhaps the most remarkable aspect of the TWR solutions, even though the detectable variation is only at the $30 \%$ level.

Naturally, our solution for $\Omega_{p}(r)$ lends itself to pattern winding time estimates. With the average values for the maximum and minima implied by our solution, we can estimate the winding time of the pattern according to

$$
\tau_{\text {wind }}=2 \pi /\left(\Omega_{p, \max }-\Omega_{p, \min }\right) .
$$

For the outer spiral arm, for example, we estimate an average time to wind $\bar{\tau}_{\text {wind }}=71.64$, which is less than $10 \%$ from the actual winding time $\tau_{\text {wind }}=78.53$ observed from the time evolution of the simulation. [This, of course, assumes that $\Omega_{p}(r)$ does not vary over this time.]

As this simulation would indicate, even without uniform slice coverage, although it may be slightly more difficult to determine with confidence the radial domain of the pattern (given large errors in $r_{t}$ and $r_{c}$ ), the overall shape, or functional form, for $\Omega_{p}(r)$ can be ascertained. Of course, this is largely influenced by the adopted measurement errors $\sigma^{\langle v\rangle}$ for each slice and the quality of a priori information that can be gathered and employed. With larger errors $\sigma^{\langle v\rangle}$, for instance, the $\chi^{2}$ criterion becomes less discriminating, and it may be difficult to distinguish between several different radial dependencies for $\Omega_{p}(r)$. In addition, without clear evidence that limits where the spiral pattern terminates, we risk misidentifying intrinsic radial variation. Indeed, if we restrict $r_{c}$ to less than that implied by the best-fit solutions and search instead for solutions at a second $r_{c}-\chi^{2}$ minimum, then the pattern speed solutions for all six SAs are constant between $\bar{r}_{t} \sim 0.47$ and $\bar{r}_{c} \sim 2.47$. As may be expected, the average value for this constant pattern speed $\Omega_{p}=0.236 \pm 0.051$ is similar to that suggested by traditional TW estimates, where $\Omega_{p, \mathrm{TW}}=0.207 \pm 0.046$ on average.

\subsection{Simulation III: Double-barred Galaxy}

In this section we address the use of the TWR method for the purposes of nuclear bar detection and measurement using the double-barred SB0 simulation pictured in Figure 18. In performing the regularized TWR calculation, we again act under the assumption of multiple patterns in distinct radial zones. Our models for $\Omega_{p}(r)$ parameterize unique, constant pattern speeds for both the primary and secondary bars, known to have pattern speeds of $\Omega_{\mathrm{pb}}=0.23$ and $\Omega_{\mathrm{sb}}=0.41$, respectively. From inspection of the surface density and its Fourier decomposition (for which the power spectrum is plotted in Fig. 19), we associate the drop in power of 

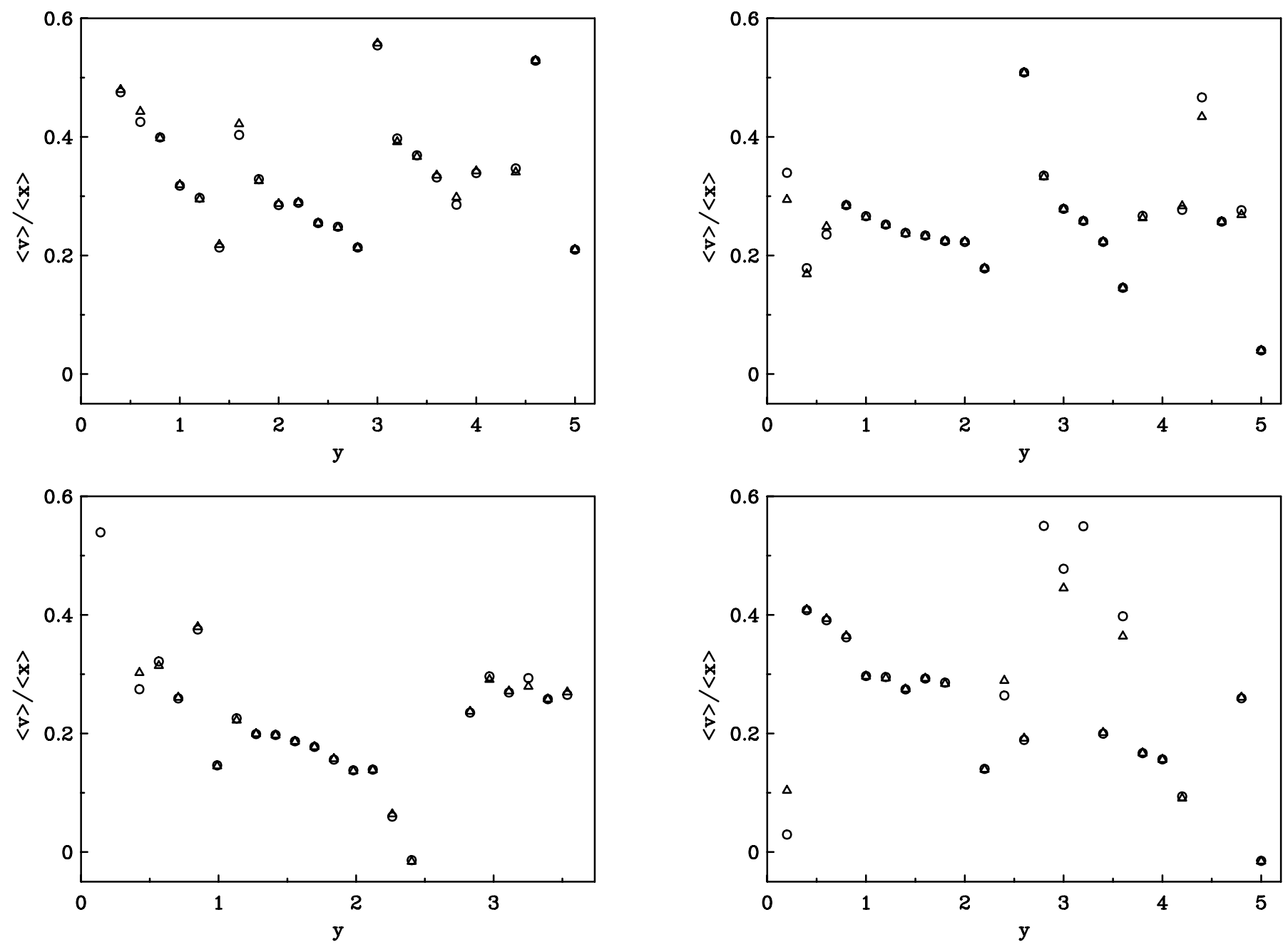

FIG. 16.-Plot of $\Omega_{p}=\langle v\rangle /\langle x\rangle$ as a function of distance from the galaxy major axis $y$ for the spiral simulation at the four SAs in Fig. 15 (clockwise from top left: $45^{\circ}$, $15^{\circ},-45^{\circ}$, and $\left.-15^{\circ}\right)$. Triangles (circles) mark slices on the $y>0(y<0)$ side of the galaxy.

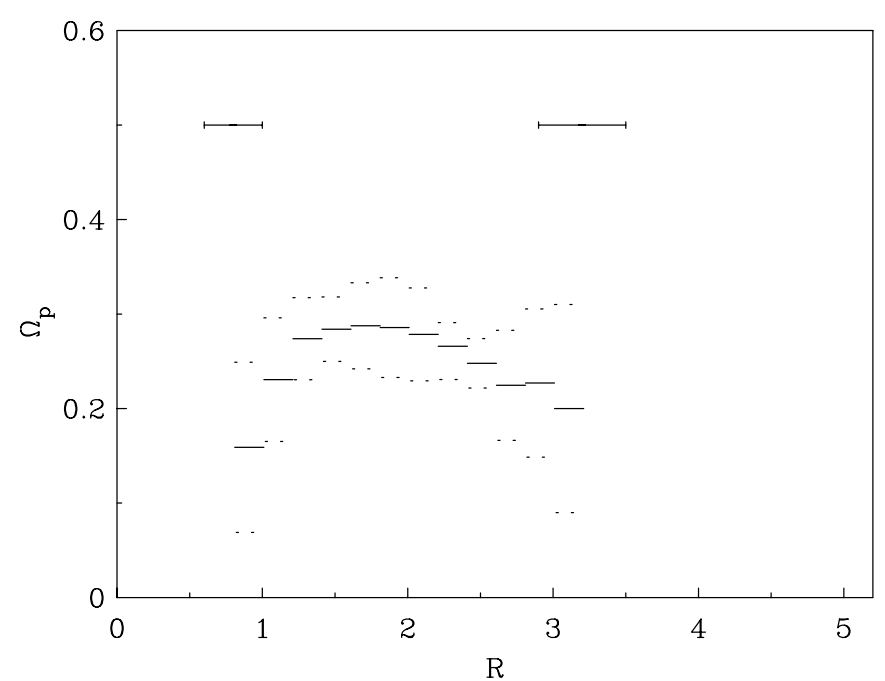

FIG. 17.-Best-fit regularized solution and error bars from the TWR method using $\Delta r=0.2$ bins as applied to the spiral simulation averaged over six SAs. The best solution is shown as a series of solid lines for each bin with dashed errors. Errors for the transitions $r_{t}=0.8 \pm 0.2$ and $r_{c}=3.2 \pm 0.3$ are represented by horizontal error bars at the top.

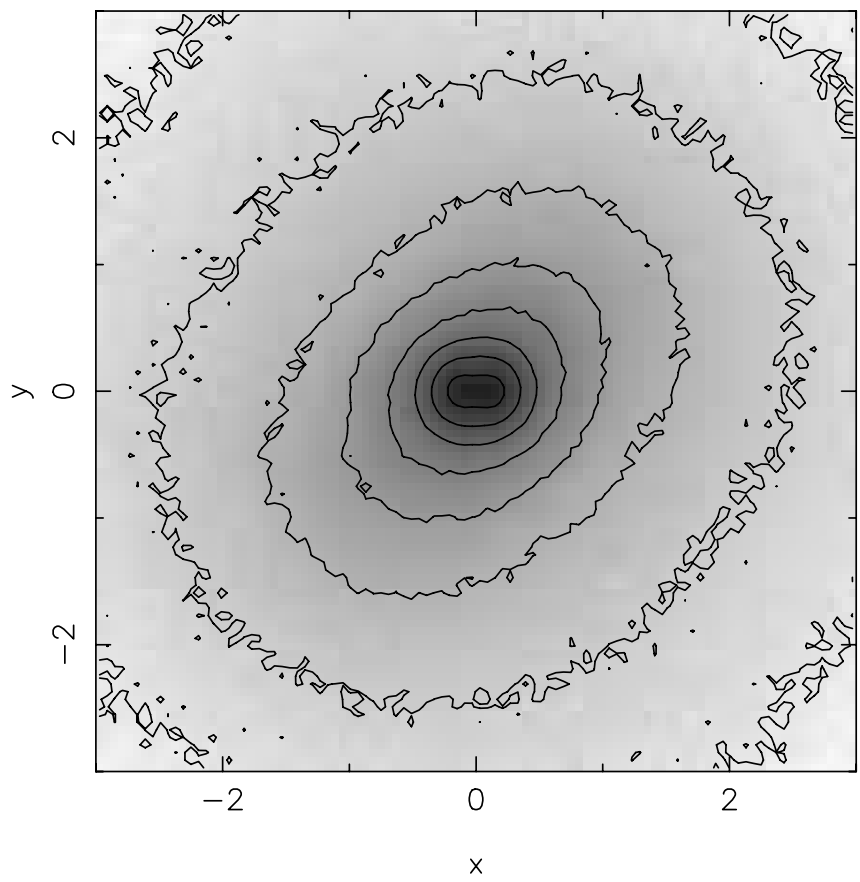

FIG. 18.-Face-on display of the double-barred simulation's surface brightness distribution projected without rotation about the $z$-axis, highlighting the inner two bars. 


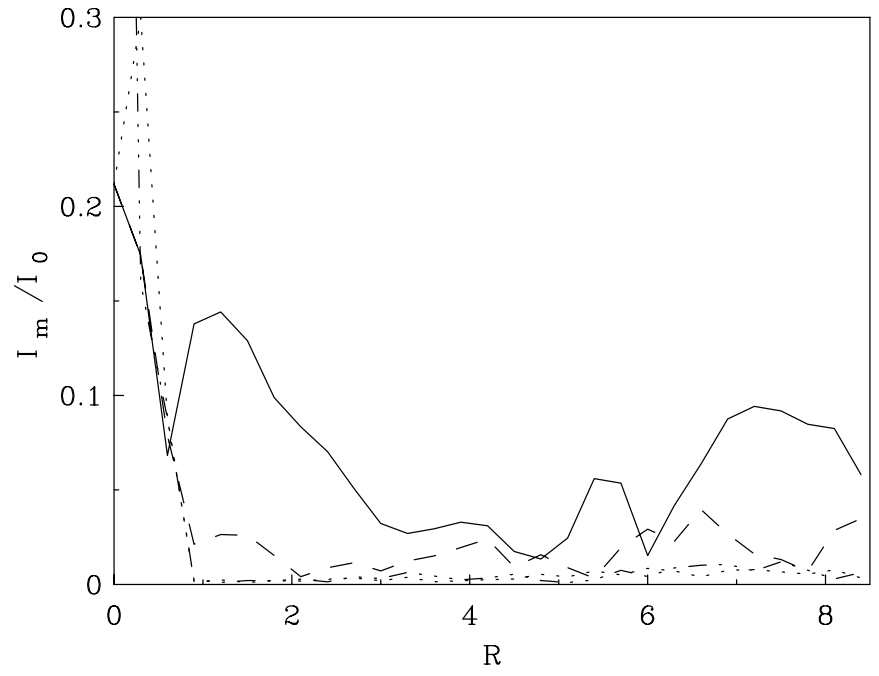

FIG. 19.-Fourier power spectrum of the double-barred simulation's surface brightness distribution shown in Fig. 18. Modes up to $m=4$ are plotted as a function of radius: $m=1$ (dotted line), $m=2$ (solid line), $m=3$ (triple-dot-dashed line), and $m=4$ (dashed line).

the $m=2$ component at $r \sim 0.8$ and again at $r \sim 3.0$ with the end of each bar. In step models for $\Omega_{p}(r)$, then, we restrict the secondary bar-to-primary bar and primary bar-to-disk transitions to within $0.4<r_{t, 1}<1.2$ and $2.5<r_{t, 2}<3.2$, respectively.

To isolate the bars from the rest of the disk, we extend the quadrature to the edge of the surface density and employ our cut procedure in light of the argument set forth at the end of $\S$ 4.2.2. This is particularly compelling here since, despite the apparent axisymmetry beyond the primary bar in this SB0 simulation, the Fourier decomposition shows power in the $m=2$ mode beyond $r \sim 3.0$, especially in the last third of the disk. If the asymmetry in this radial zone (which appears only very weakly in the surface density) is sustained by an ill-defined, nonunique, or perhaps unrigid pattern, for which the TW/TWR assumptions break down, then associating it with a measurable pattern speed will likely introduce regularization-induced bias to the solution for the interior patterns of interest.

Critically, asymmetry such as this may prevent clear integral convergence beyond the primary bar end. So, too, can its presence in the integrals be expected to degrade the reliability of pattern speed estimates for the structures of interest. Removing the influence of the information in this outer radial zone by calculating the bins there without regularization is our best chance for accurate pattern speed measurement. Given that the departure from axisymmetry manifest by a small nuclear bar will be relatively minor compared with that of other patterns in the disk, this is especially relevant for accurate measurement of $\Omega_{s}$. In this case, nonaxisymmetry on a comparable scale may easily upset this structure's contribution to the integrals and, if prescribed an incorrect pattern speed model, may introduce consequences for the innermost bins in solutions.

Our procedure for this simulation, however, does not quite involve calculating without regularization all bins up to the patterns of interest (i.e., to the end of the primary bar). As in all cases, but particularly here where we are compelled to cut approximately two-thirds of the disk, leaving a large portion of the total bins unregularized may begin to reintroduce unamendable propagating noise. To reduce this risk, careful attention has been paid to the development and testing of models that prevent the destabilization of solutions. [Indeed, the appropriate balance between noise and stability in models for $\Omega_{p}(r)$ must be explored on a galaxy-by- galaxy basis.] Here we find that the most stable and realistic models for $\Omega_{p}(r)$ are those that include a third, constant pattern past the end of the primary bar. From this we might infer that, although weak and difficult to discern in Figure 18, there exists a spiral pattern outside the primary bar, perhaps corresponding to the $m=2$ component beyond $r \sim 3.0$, which remains clear but modest out to $r \sim 5.0$. Indeed, we assume that the third minimum in the power of the $m=2$ component at this radius corresponds to the end of the spiral pattern and, moreover, since counts are low in the rest of the disk, that the bins beyond $r \sim 5.0$ are best calculated without regularization. We note, however, that we do not necessarily expect to measure a realistic pattern speed in this third radial zone.

Compiling this evidence for two bars and a possible spiral, we search for the best-fit solutions parameterizing two constant pattern speeds (one for the primary bar, one for the inner secondary bar) out to the end of the primary bar, in addition to a third constant pattern speed $\Omega_{s}$ restricted to extend out to $4.3<r_{c}<5.3$. We find that solutions generated in this manner provide much more accurate estimates for the primary and secondary bars compared with solutions that are either regularized over the full extent of the surface density or unregularized up to the end of the primary bar.

For the purposes of further establishing favorable conditions for nuclear bar detection, we adopt a small bin width $\Delta r=0.15$ in the quadrature. As described in $\S \S 4.2 .1$ and 4.2.4, the mismatch by a fraction of a bin width or more between the actual transition and that to which the solution is confined can have consequences for both inner and outer TWR pattern speed estimates. Real nuclear bars will need to be well resolved in order to accurately separate the contributions of the two bars.

The secondary bar in this simulation is known to be nonrigidly rotating; in Shen \& Debattista (2007) and Debattista \& Shen (2007) the amplitudes and pattern speeds of secondary bars formed in purely collisionless $N$-body simulations through the introduction of a rotating pseudobulge oscillate as the bars rotate through the companions in which they are nested. In Shen \& Debattista (2007) TW estimates of the secondary bar pattern speed measured using bulge-only kinematics (the bulge supports the nuclear bar alone and a primary bar contribution need not be accounted for in the TW integrals) are subject to marked errors consistent with an origin in nonrigid rotation. These errors result in estimates of $\Omega_{\mathrm{sb}}$ too high on one side of the galaxy and too low on the other, in accord with being a manifestation of the oscillations driving radial pulsations that contribute with different signs on the two sides of the galaxy. Cancellation between measurements from both sides of the galaxy in global regularized solutions reduces the effect of the oscillations. In the discussion to follow, our focus is on sources of error in the TWR calculation other than this intrinsic effect.

The TWR estimates from solutions with $\Delta r=0.15$ bins at six SAs $\left( \pm 15^{\circ}, \pm 45^{\circ}\right.$, and $\left.\pm 75^{\circ}\right)$ are listed in Table 6 , and the average and rms for this SA range are shown in Figure 20. Compared with solutions generated using $\Delta r=0.3$ bins in the calculation, these solutions are much more stable and accurate; at less than $8 \%$ from their actual values, the comprehensive primary and secondary bar pattern speeds evidently benefit from the integrity of the calculated transitions between patterns (we find $r_{t, 1}=0.75 \pm$ $0.09, r_{t, 2}=3.0 \pm 0.21$, and $\left.r_{c}=4.6 \pm 0.33\right)$.

Although the TWR estimates in Figure 20 are accurate, the rms in each suggests $8 \%-13 \%$ error on the part of the method. In addition to the intrinsic nonrigid rotation error expected for the secondary bar measurement, this may yet indicate the slight misdesignation of transitions between patterns, and we can also assume that (although we do not necessarily consider its measurement 
TABLE 6

TWR Estimates for SimUlation III

\begin{tabular}{|c|c|c|c|c|c|c|}
\hline $\begin{array}{c}\text { SA } \\
\text { (deg) }\end{array}$ & $\Omega_{\mathrm{sb}}$ & $\Omega_{\mathrm{pb}}$ & $\Omega_{s}$ & $r_{t, 1}$ & $r_{t, 2}$ & $r_{c}$ \\
\hline $75 \ldots$. & 0.420 & 0.231 & 0.146 & 0.75 & 3.15 & 4.35 \\
\hline ........................ & 0.548 & 0.278 & 0.178 & 0.75 & 3.15 & 4.35 \\
\hline $15 \ldots$ & 0.407 & 0.231 & 0.063 & 0.6 & 2.7 & 4.8 \\
\hline ….............. & 0.404 & 0.264 & 0.087 & 0.75 & 2.7 & 5.25 \\
\hline$-45 \ldots \ldots \ldots \ldots \ldots \ldots \ldots$ & 0.477 & 0.259 & 0.233 & 0.9 & 3.15 & 4.35 \\
\hline$-75 \ldots \ldots \ldots \ldots \ldots \ldots \ldots$ & 0.375 & 0.225 & 0.028 & 0.9 & 3.15 & 4.5 \\
\hline Actual ............................ & 0.41 & 0.23 & $\ldots$ & 0.8 & 3.0 & 5.0 \\
\hline
\end{tabular}

NOTES.-The secondary bar, primary bar, and spiral pattern speeds listed here are estimated from TWR solutions calculated using a $\Delta r=0.15$ bin width for a range of SAs. The last three columns list the connate estimates for $r_{t, 1}, r_{t, 2}$, and $r_{c}$. Values for the actual pattern speeds are shown in the last row.

realistic) the third measured pattern speed has a nontrivial influence on the inner pattern speeds, as must $\Omega_{\mathrm{pb}}$ on $\Omega_{\mathrm{sb}}$, for instance.

With so many free parameters in our model (more than in any other used in this paper), identifying unequivocally the dependence of each on the others is difficult (indeed, the rms in each estimate likely reflects the combined consequence of many such interdependencies). As in previous sections, however, we can begin to understand the largest source of variability in the estimates from SA to SA by considering the influence of morphology and slice orientation on the solutions. If we consider that the major axes of the two bars are oriented at $\sim 45^{\circ}$ from one another at this time step (that is, with the secondary bar aligned along the $x$-axis, the primary bar is aligned along the line $y=x$, or in the Shen \& Debattista [2007] convention, $\Delta \phi=45^{\circ}$ ), then slices at $\pm 45^{\circ}$ are oriented along the primary bar minor/major axis. In this case, even in the presence of the secondary bar, $\langle v\rangle$ and $\langle x\rangle$ for the inner primary bar crossing slices fluctuate noisily about zero.

This behavior is more than enough to prevent accurate TW estimates for the primary bar (which Rand \& Wallin [2004] find are less reliable when bars are aligned within $\pm 20^{\circ}$ of a principle axis), and so it is perhaps not surprising that our TWR estimates at $\pm 45^{\circ} \mathrm{SAs}$ show rather large inaccuracies. However, what we find requires slightly more interpretation: it is the secondary bar estimate, and not $\Omega_{\mathrm{pb}}$, that is compromised.

Unlike the results in $\S 4.2$, with which we could argue that the regularized TWR method is, in principle, fairly equipped to accommodate any alignment of patterns (and where even slices at $\pm 15^{\circ}$ from the bar axis return accurate TWR estimates), here we are not only dealing with more model parameters (and their intrinsic covariance), but the innermost pattern now has a much smaller extent than the structures in the disk. Consequently, the secondary bar occupies the smallest fraction of bins in the innermost slices where, moreover, the errors $\sigma_{i}$ are the largest. Presumably, in this exploitable state the secondary bar estimate is sacrificed for the primary bar $\Omega_{\mathrm{pb}}$ in the regularized calculation; at $\mathrm{SA}= \pm 45^{\circ}$, our measurement of $\Omega_{\mathrm{sb}}$ is overestimated by $\sim 25 \%$, while $\Omega_{\mathrm{pb}}$ is good to within $11 \%$, on average. (We can assume that such imprecision also reflects the effect of the secondary bar's nonrigid rotation.) At other slice orientations where the primary bar is more favorably sampled, the trade-off is less severe (although modestly PA dependent).

An impromptu calculation using particles in the bulge only (motivated by the Shen \& Debattista [2007] strategy) seems to confirm the influence of the primary bar in the TWR calculation at $\mathrm{SA}=45^{\circ}$. The error in our lone estimate $\Omega_{\mathrm{sb}}=0.42$ in this case is significantly less than when the primary bar is present and

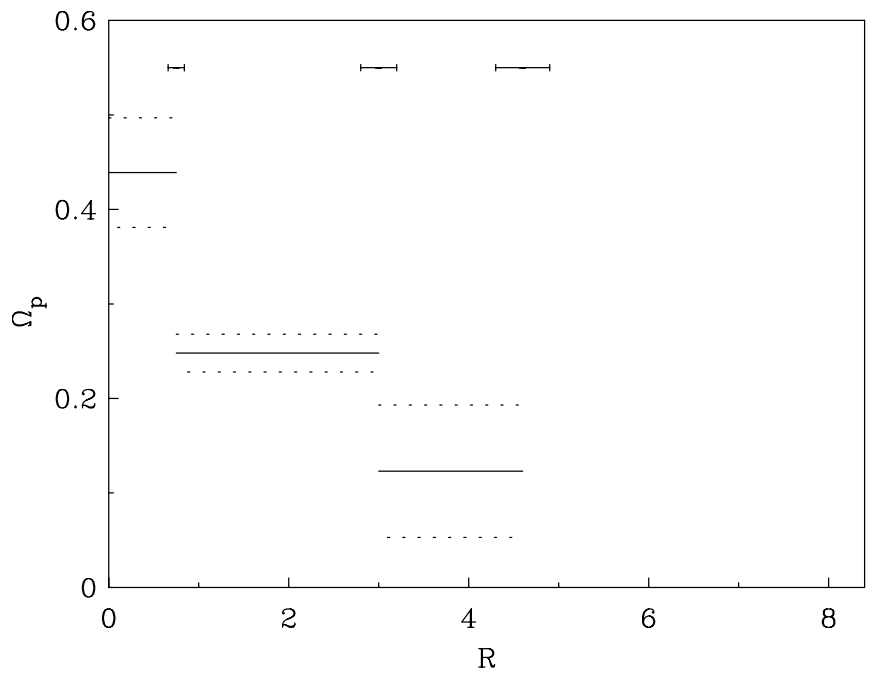

FIG. 20.-Best-fit solution and error bars from the TWR method as applied to the double-barred simulation averaged over six SAs using $\Delta r=0.15$ bins. The secondary bar $\Omega_{\mathrm{sb}}=0.439 \pm 0.058$, primary bar $\Omega_{\mathrm{pb}}=0.248 \pm 0.02$, and spiral $\Omega_{s}=0.123 \pm 0.07$ are shown as solid lines with dashed errors. Horizontal error bars at the top indicate the dispersion in the secondary bar-to-primary bar transition $r_{t, 1}=0.75 \pm 0.09$, the primary bar-to-spiral transition $r_{t 2}=3.0 \pm 0.21$, and spiral termination radius $r_{c}=4.6 \pm 0.33$.

also comparable to that in bulge-only estimates at other SAs; for the three SAs in quadrant II tested, we find $\Omega_{\mathrm{sb}}=0.43 \pm 0.01$.

Even if the TWR method cannot accurately measure the pattern speed of a pulsating nuclear bar (aside from with global regularized solutions, in particular), it is nevertheless appropriate for use in characterizing and constraining $\Omega_{\mathrm{sb}}$, especially in the presence of a primary bar. The comparative worth of TWR solutions can be deduced in light of the distinction between the traditional TW and TWR methods. Our TWR pattern speed measurement for the primary bar is comparable to that from the traditional TW calculation; using slices covering $|y| \leq 2.25$ (such that the bar contribution is maximal in all slices), the TW method returns $\Omega_{\mathrm{pb}, \mathrm{TW}}=0.214 \pm 0.011$. The TWR secondary bar estimate, on the other hand, greatly improves on the TW estimate $\Omega_{\mathrm{sb}, \mathrm{TW}}=$ $0.338 \pm 0.149$ available using slices covering $|y| \leq 0.6$.

Since the nuclear bar appears in only a very small number of slices and its contribution is, moreover, easily overwhelmed in the extended TW integrals, the TW method only modestly recovers $\Omega_{\mathrm{sb}}$. In the TWR calculation, however, the few bins that cover the nuclear bar are supplied with large weights $K_{i j}$ compared with the rest of the information along each slice. Together with a suitable bin width, this effectively isolates information from throughout the nuclear bar extent from that of all other patterns in the disk. The quality with which $\Omega_{\mathrm{sb}}$ can be constrained then depends on how well the contribution from these other patterns is identified and removed from slices intersecting the nuclear bar; moreover, it relies on regularization over both sides of the galaxy to construct a global solution.

Aside from the unavoidable error introduced by the nonrigid rotation of nuclear bars expected from $N$-body simulations, from our current study it seems likely that similar calculations may be limited according to the degree of resolution and the relative size of the secondary bar. That is, the former dictates the precision with which the secondary bar-to-primary bar transition can be determined, and the latter sets the leverage supplied by the secondary bar to the $\chi^{2}$. Furthermore, given that the measurement of an inner, nuclear bar pattern speed is subsequently largely affected by how well the other patterns present in the disk can be 
measured, success with the TWR method requires appropriate models for $\Omega_{p}(r)$ that account for such concerns.

\section{SUMMARY}

\subsection{Caveats in Applications to Real Galaxies}

The successes of the TWR method as applied to simulated data should be obtainable with real galaxies, provided that adequate attention is paid to several considerations. Since the calculation foremost requires integration along slices parallel to the galaxy major axis that reflect information from all patterns of interest in the disk, observations must be able to present a number of these. As governed by the resolution or sampling of the data, the placement of these slices must be able to define a quadrature wherein the position $y$ of the outermost slice corresponds to that of the last matrix element along the slice requiring the largest limit of integration $X_{\max }$ for integral convergence; when convergence can be reached only clearly at the map boundary (perhaps far beyond the extent of the patterns), this is essential for correctly accounting for all information along each slice.

Establishing the slice positions and orientation for an accurate quadrature also clearly requires superior knowledge of kinematic and morphological parameters. In general, the same restrictions to the quality of input data in traditional TW calculations apply to the TWR method. As described in $\S 4.2 .3$, errors in the assumed PA that impair TW estimates can also introduce considerable inaccuracy to the TWR solutions. The inclination angle of the disk must also be well determined and should be preferably restricted to only moderate values (which, beyond the observational requirements of the TW method, will keep errors from corrupting the association of information into accurate radial bins).

Additionally, although more easily overcome in traditional TW estimates (Merrifield \& Kuijken 1995), errors in the systemic velocity and galaxy center might prove critical for the TWR solutions since each side of the disk provides an independent solution for $\Omega_{p}(r)$. Care must be taken not to impair the prevailing symmetry along each slice, given that each integral bears more than one estimate. The incorrect placement of radial bins according to a misassigned galaxy center position, for example, could significantly over- or underestimate the actual pattern speed and moreover make assessment of the true radial variation unlikely.

The regularization procedure developed here itself makes further demands on the quality and amount of information necessary to perform the calculation. But by keeping the amount of information beyond that required of the TW method to a minimum, and using standard diagnostics such as Fourier decomposition, the requisite set of a priori assumptions can be invoked quite reliably. As long as the information is accessible, requiring at the least theoretical motivation to develop testable models, and limited in principle only by the quality of information from which it is to be gathered, then the regularization should proceed without impediment.

Of course, unlike the simulated galaxies studied here where there is plenty of signal throughout the disk, observations of real galaxies may present sensitivity issues. While regions of low signal-to-noise ratio in the outer disk can be superseded using the cut procedure developed here, high-quality information from the rest of the disk is an obvious priority for the method; the departures from axisymmetry induced by all patterns present in the disk must be clearly detectable. Not only does the calculation depend on the presence (or lack) of these signatures, in both the surface density and the velocity field, but the mere identification of the number and domain of patterns is critical for developing appropriate models for $\Omega_{p}(r)$.
The latter necessity may be hard met since, for instance, it will be rare to observe galaxies with surface densities that can be Fourier-decomposed as cleanly as is possible with simulated galaxies. Furthermore, unlike simulations, it is impossible to establish whether or not there exists more than a single pattern speed at each measured radius in real galaxies. Since the models developed with regularization here are incompatible with nonunique pattern speeds, for real galaxies, the choice of when and where to consider a transition or to keep solutions unregularized may be based on a more qualitative assessment of where clear structure ends.

Inevitably, the combination of the above considerations (related, overall, to the quality of the data) will determine the extent to which the model for the true radial behavior of $\Omega_{p}(r)$ can be differentiated from other models. That is, the $\chi^{2}$ criterion with which we judge the goodness of solutions becomes less discriminating the larger the measurement error $\sigma^{\langle v\rangle}$. Since the adopted measurement error $\sigma^{\langle v\rangle}$ for each slice used in the calculation and in the $\chi^{2}$ estimator must necessarily incorporate observational errors based on random noise in the data, with severe enough errors different model solutions from real, imperfect data may be indistinguishable.

In addition, systematic errors (likely dominated by PA uncertainty) will undeniably challenge the accuracy of solutions. In all applications of the method it is critical to assess the influence of these errors through direct tests of the sensitivity of solutions to departures from the nominal values of PA, inclination, and kinematic center, for instance. Clearly, this makes 2D coverage desirable; here $2^{\circ}-3^{\circ}$ uncertainties in the PA alone are shown to introduce around $15 \%$ error in measurements of $\Omega_{p}(r)$ for the barred spiral simulation.

Insufficient resolution or sampling may also impair TWR solutions from real galaxies. A large adopted bin width not only limits the detectable radial variation in $\Omega_{p}(r)$ but also restricts how well multiple patterns can be separated in the resultant quadrature; a mismatch between the actual transition and that to which the solution is confined can have consequences for the estimates of both inner and outer patterns. Naturally, depending on the models to be tested and relative size of the disk, a resolutionconstrained bin width is not guaranteed to impair solutions for all galaxies. We nonetheless foresee that the only true way to preserve the integrity of solutions is with high-resolution observations.

Data cubes lend themselves well to analysis with the TWR method, since, unlike long-slit spectroscopic observations, the galaxy PA, inclination, systemic velocity, and (kinematic) center can be derived with errors from the data using a tilted ring analysis on the first moment of the cube. In addition, multiple slices can be defined with a single observation. So between radio and submillimeter investigations of spiral structure, for instance, and IFU spectroscopy, with which double-bar systems (and eventually double-barred spirals, given larger, more sensitive IFUs) can be studied, applications of the TWR method could be extensive.

Of course, like all applications of the TW method, the observed tracer must be found to obey continuity and the relation between the intensity of the tracer and the surface density must be linear or well determined everywhere. Reviews of several possible tracers argued to suitably obey the TW continuity requirement can be found throughout the literature, but we note here that the work of Gerssen \& Debattista (2007) studying the effect of dust on TW measurements of bars may find meaningful extension in future TWR studies of multiple patterns in late-type galaxies. There, model dust lane features associated with bars introduce errors on the order of $20 \%-40 \%$ (Gerssen \& Debattista 2007). In addition to these errors, TWR solutions could possibly 
be prone to increased error from spiral dust lanes at larger radii. Although it is beyond the scope of this work to make a detailed assessment of the sensitivity of TWR solutions to dust, we argue that such noise could well be mitigated through the use of regularization and expect no greater effect in TWR measurements than in TW, which, moreover, will be apparent with the use of only optical tracers.

In the immediate future, we plan to apply the TWR method to several high-resolution BIMA SONG CO observations of molecule-dominated galaxies to search for spiral winding, relations between bar and spiral pattern speeds, and spiral-spiral mode coupling. (These observations include single-dish data and therefore do not suffer from missing flux, which would be a violation of the continuity requirement.) For those galaxies with interstellar media not dominated by molecular gas, we plan to combine the $\mathrm{CO}$ with $\mathrm{H}$ I data to make total column density maps (assuming that the ionized component is negligible). Since the $\mathrm{CO}-\mathrm{H}_{2}$ conversion factor is critical in combining the $\mathrm{CO}$ and $\mathrm{H}$ I maps (and, of course, in establishing molecule dominance in the former case), it will be necessary to test the sensitivity of the TWR method to the adopted conversion factor for such combinations.

In addition, since warped disks (common in $\mathrm{H} \mathrm{I}$ ) are a violation of the TW assumptions, we will also perform tests to determine if our cutoff scheme can be used to circumvent the warp and thereby extract solutions from the rest of the disk. For these applications of the TWR method, we plan to construct measurement errors $\sigma$ for each slice that reflect uncertainties related to the flux cutoff chosen in creating the moment maps. The effect of PA and other systematic errors will be assessed by testing the sensitivity of solutions to departures from the nominal values.

\subsection{Applying the TWR Method}

Although in the interest of testing our strategy for each simulated galaxy is somewhat tailored to its unique properties, with the above caveats in mind our studies have enabled us to develop a general and reasonably objective prescription for applying the regularized TWR calculation:

1. Establish the bin width and the corresponding number of slices (not necessarily uniformly spaced) that are required to achieve converged integrals using an $N \times N$ quadrature. For the purposes of measuring multiple patterns in a single disk, this will likely extend to the map boundary.

2. Compile a priori information by inspecting the surface density, its Fourier decomposition, and the velocity field for indications of patterns and to establish the expected number and domain of measurable pattern speeds. This should include the identification of regions in the disk susceptible to regularization-induced bias.

3. Develop theoretically and/or observationally motivated models that parameterize $\Omega_{p}(r)$ according to the a priori information.

4. Incorporate measurement errors into a single $\sigma^{\langle v\rangle}$ (and $\sigma$ ) for each slice. These should represent uncertainties in the adopted intensity noise level and/or other random noise-related errors; systematic errors are preferably determined through direct testing (see item 9).

5. Develop the weighting scheme for a reduced $\chi^{2}$ estimator that accounts for the total degrees of freedom for the models to be tested. This should reflect expectations for which slices, if any, are most critically to be reproduced by the models.

6. Generate preliminary solutions for the models. At this point, the degree of regularization required to return solutions according to type should be explored.
7. With finalized solutions, use equation (7) to generate a complete set of $\langle v\rangle$ for each and calculate the corresponding $\chi_{\nu}^{2}$.

8 . Use the $\chi_{\nu}^{2}$ to identify the best-fit solution.

9. Test the sensitivity of the results to other systematic effects peculiar to the observation, e.g., adopted PA and/or the CO- $\mathrm{H}_{2}$ conversion factor, for instance.

\section{CONCLUSIONS}

In this paper we have shown that regularizing the TWR calculation is an effective means of smoothing intrinsically noisy solutions for more precise measurement of $\Omega_{p}(r)$. Specifically (barring a large, limiting resolution), regularization admits the use of a much smaller bin width than that required to achieve comparable smoothness in the unregularized calculation. This affords improved assessment of radial variation, as well as more accurate determination of the transitions between multiple pattern speeds (and thus of the values of the pattern speeds themselves, in principle). Moreover, with the regularized TWR calculation, different theoretically and observationally motivated models for the radial dependence of $\Omega_{p}(r)$ can be tested in fairly short time and with only the minor addition of information compared to the unregularized TWR and TW methods.

With a simple scheme for generating $n$ th-order polynomial solutions that can be incorporated into step models, we have shown that the TWR method can be used to parameterize the radial domains of multiple pattern speeds. Together with a priori information identifying zones in the disk that may be incompatible with measurement (because they either are characterized by low signalto-noise ratio or show no evidence for a pattern), we can further constrain the extent of patterns while optimally reducing regularization-induced bias in pattern speed solutions.

As applied to three simulated galaxies, we find that the TWR method developed in this manner performs with a high degree of accuracy (with less than $15 \%$ error) both in measurement and in extracting information about the true functional form for the pattern speed. Tests on a simulation of a barred spiral galaxy indicate that not only can the constant pattern speed for a relatively weak spiral be reliably reproduced, but information about both the pattern speed and the radial extent of the bar pattern can also be extracted. (Indeed, we find that the bar pattern speed estimate is strengthened by the proper use of information from beyond the bar end.) And although the bar pattern speed estimates are highly susceptible to systematic errors (with PA errors introducing the largest uncertainty to TWR pattern speeds, as with TW estimates), we find that the identification of the transition between the two is relatively stable.

The TWR method can also be effectively employed to measure patterns that are winding in nature. In a simulation of a two-armed spiral, the best-fit TWR solutions from several slice orientations correctly reproduce the high-order radial variation of the pattern speed, despite modest indication that not all orientations supply the same authority (this, of course, would seem to depend on the morphology of this spiral, in particular). Indeed, although the TWR method can, in principle, handle any (presumably random) alignment of patterns, in all of the simulations studied, slice orientations that provide the most uniform coverage of the patterns are preferred. This is of particular importance for nuclear bar pattern speed measurement, as found in tests of the method on a doublebarred simulation. Since the innermost bins that provide the foremost leverage on the nuclear bar are also the most susceptible to errors from throughout the disk, confident measurement requires all other patterns to be well constrained. 
In principle, comparable accuracy should be achievable on real galaxies. However, these tests do not constrain how well the TWR method can perform under severe observational limitations that may commonly arise. Not only can determinations of the PA, inclination, and dynamical center be subject to considerable errors given low-quality data, but identifying constraints on the patterns present in the disk to be incorporated into models for $\Omega_{p}(r)$ could prove challenging. In addition, although regularization can reduce the impact of noise on solutions, large measurement errors for each slice could make discriminating between several possible models for $\Omega_{p}(r)$ difficult. And most critically, since the nature of the numerical calculation relies on a relatively small bin width to achieve its greatest accuracy, without high resolution, some observations may not afford practical solutions.

Nevertheless, if restricted to high-resolution observations with adequate sensitivity, and given radially stable kinematic parameters, TWR solutions can be used to study the connection between multiple patterns and the nature of spiral winding. So, too, can we expect progressively more satisfactory applications of the method; although the number of galaxies to which the method can be successfully applied is limited by the current generation of instruments, in the future, larger IFUs, ALMA, and eventually SKA should yield much higher quality data with larger areal coverage and higher angular resolution. This prospect in itself should warrant future studies with the TWR method.

This research was supported in part by NASA through the American Astronomical Society's Small Research Grant Program. S. E. M. acknowledges support from a NASA-funded New Mexico Space Grant Consortium Graduate Research Fellowship. V. P. D. is supported by an RCUK Fellowship at the University of Central Lancashire. J. S. acknowledges support from a Harlan J. Smith fellowship. This material is based on work partially supported by the National Science Foundation under grant AST 03-06958 to R. J. R.
Corsini, E. M., Debattista, V. P., \& Aguerri, J. A. L. 2003, ApJ, 599, L29

Debattista, V. P. 2003, MNRAS, 342, 1194

Debattista, V. P., Corsini, E. M., \& Aguerri, J. A. L. 2002a, MNRAS, 332, 65

Debattista, V. P., Gerhard, O., \& Sevenster, M. N. 2002b, MNRAS, 334, 355

Debattista, V. P., Mayer, L., Carollo, C. M., Moore, B., Wadsley, J., \& Quinn, T. 2006, ApJ, 645, 209 (D06)

Debattista, V. P., \& Sellwood, J. A. 1998, ApJ, 493, L5 2000, ApJ, 543, 704

Debattista, V. P., \& Shen, J. 2007, ApJ, 654, L127

Debattista, V. P., \& Williams, T. B. 2004, ApJ, 605, 714

Elmegreen, B. G., Elmegreen, D. M., Chromey, F. R., Hasselbacher, D. A., \& Bissell, B. A. 1996, AJ, 111, 2233

Elmegreen, B. G., Elmegreen, D. M., \& Seiden, P. E. 1989, ApJ, 343, 602

Fathi, K., Toonen, S., Falcon-Barroso, J., Beckman, J. E., Hernandez, O., Daigle, O., Carignan, C., \& de Zeeuw, T. 2007, ApJ, 667, L137

Garcia-Burillo, S., Combes, F., \& Gerin, M. 1993, A\&A, 274, 148

Gerssen, J., \& Debattista, V. P. 2007, MNRAS, 378, 189

Kormendy, J., \& Norman, C. A. 1979, ApJ, 233, 539

Maciejewski, W. 2006, MNRAS, 371, 451
REFERENCES

Masset, F., \& Tagger, M. 1997, A\&A, 318, 747

Merrifield, M. R., \& Kuijken, K. 1995, MNRAS, 274, 933

Merrifield, M. R., Rand, R. J., \& Meidt, S. E. 2006, MNRAS, 366, L17 (MRM06)

Miller, K. 1970, SIAM J. Math. Anal., 1, 52

Rand, R. J., \& Wallin, J. F. 2004, ApJ, 614, 142

Rautiainen, P., \& Salo, H. 1999, A\&A, 348, 737

- 2005, ApJ, 631, L129

Roberts, W., \& Stewart, G. 1987, ApJ, 314, 10

Sellwood, J. A., \& Kahn, F. D. 1991, MNRAS, 250, 278

Sellwood, J. A., \& Lin, D. N. C. 1989, MNRAS, 240, 991

Sellwood, J. A., \& Sparke, L. S. 1988, MNRAS, 231, 25P

Shen, J., \& Debattista, V. P. 2007, ApJ, submitted (arXiv:0711.0966)

Sygnet, J. F., Tagger, M., Athanassoula, E., \& Pellat, R. 1988, MNRAS, 232, 733

Tikhonov, A. N., \& Arsenin, V. Y. 1997, Solutions of Ill-posed Problems (New York: Wiley)

Tremaine, S., \& Weinberg, M. D. 1984, ApJ, 282, L5

Zimmer, P., Rand, R. J., \& McGraw, J. T. 2004, ApJ, 607, 285 